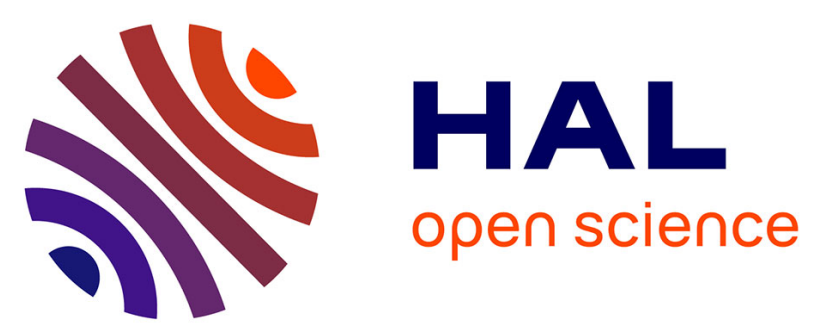

\title{
Un complexe métallurgique et minier du haut Moyen Âge: le site des Fourneaux à Vert-Saint-Denis (Seine-et-Marne)
}

\author{
Isabelle Daveau, Vincent Goustard, Jean-Jacques Bahain
}

\section{To cite this version:}

Isabelle Daveau, Vincent Goustard, Jean-Jacques Bahain. Un complexe métallurgique et minier du haut Moyen Âge: le site des Fourneaux à Vert-Saint-Denis (Seine-et-Marne). Gallia - Archéologie de la France antique, 2000, Mines et métallurgies en Gaule, 57, pp.77-99. 10.3406/galia.2000.3211 . hal-01902622

\section{HAL Id: hal-01902622 \\ https://hal.science/hal-01902622}

Submitted on 20 Jan 2020

HAL is a multi-disciplinary open access archive for the deposit and dissemination of scientific research documents, whether they are published or not. The documents may come from teaching and research institutions in France or abroad, or from public or private research centers.
L'archive ouverte pluridisciplinaire $\mathbf{H A L}$, est destinée au dépôt et à la diffusion de documents scientifiques de niveau recherche, publiés ou non, émanant des établissements d'enseignement et de recherche français ou étrangers, des laboratoires publics ou privés.

\section{(ㅇ)(1) $\$$}

Distributed under a Creative Commons Attribution - NonCommercial - NoDerivatives $\mid 4.0$ 


\title{
UN COMPLEXE MÉTALLURGIQUUE ET MINIER DU HAUT MOYEN ÂGE
}

\section{Le site des Fourneaux à Vert-Saint-Denis (Seine-et-Marne)}

\author{
Isabelle DAVEAU, Vincent GOUSTARD avec la collaboration de Jean-Jacques BAHAIN
}

\begin{abstract}
Mots-clés. Vert-Saint-Denis, Seine-et-Marne, haut Moyen Âge, mine, minerai de fer, atelier de réduction.
Key-words. Vert-Saint-Denis, Seine-et-Marne, Early Middle Ages, mining, iron ore, bloomery.

Résumé. Le complexe métallurgique du haut Moyen Âge des Fourneaux à Vert-Saint-Denis (Seine-et-Marne), associant mine, ateliers de traitement et de réduction du minerai, a pu être abordé sur une grande surface. Les différentes étapes de la transformation du minerai de fer ont été étudiées, tandis que l'habitat associé nous permet d'entrevoir le contexte socio-économique de cet artisanat.
\end{abstract}

\begin{abstract}
The melallurgical centre of the Early Middle Ages furnaces at Vert-Saint-Denis (Seine-et-Marne) combines mine, processing and smelting sites. It has been tackled on a large scale. The successive stages of transformation of iron ore has been studied while the connected settlement gives an idea of the socio-economic context of this iron industry.
\end{abstract}

Le site des Fourneaux se trouve à $4 \mathrm{~km}$ au nord-ouest du centre historique de Melun, sur le territoire de la commune de Vert-Saint-Denis, en Seine-et-Marne (fig. 28).

Sa découverte remonte à la fin des années 1960, lors de prospections de surface entreprises par l'Association pour la Carte archéologique de Melun et ses environs (ACAME). La construction, à son emplacement, du diffuseur permettant le raccordement de la nouvelle autoroute A5b sur la RN 105 a motivé la mise en place d'une opération archéologique de grande envergure. Le sauvetage urgent, financé par la Société des autoroutes ParisRhin-Rhône, s'est déroulé sur cinq mois, en 1994. Six mille structures en creux ont été mises au jour sur les 6,5 ha décapés, témoignant de l'exceptionnelle densité de ce site.

Le gisement des Fourneaux se trouve sur la bordure occidentale du plateau briard, vaste plate- forme structurale limitée à l'ouest par la vallée de la Seine et au nord par celle de la Marne. Le substratum ante-quaternaire du plateau est formé par le calcaire de Brie (Stampien inférieur), fréquemment meuliérisé et recouvert par endroits de buttes témoins de sables et grès de Fontainebleau (Stampien supérieur). Le démantèlement naturel de ces buttes sous l'effet de l'érosion a remanié considérablement le matériel sableux, en entraînant la disparition quasi totale de certaines d'entre elles. La couverture limoneuse est d'importance variable, sur le site son épaisseur est de $1 \mathrm{~m}$ à $1,50 \mathrm{~m}$.

Le gisement se développe sur le versant nord-est d'une de ces buttes stampiennes, dont le sommet culmine à $85,50 \mathrm{~m}$ NGF. Sa déclivité vers le nord est de l'ordre de $2 \%$. Le ru de Ballory, ruisseau permanent drainant cette portion du plateau briard, court à $1 \mathrm{~km}$ au nord. 


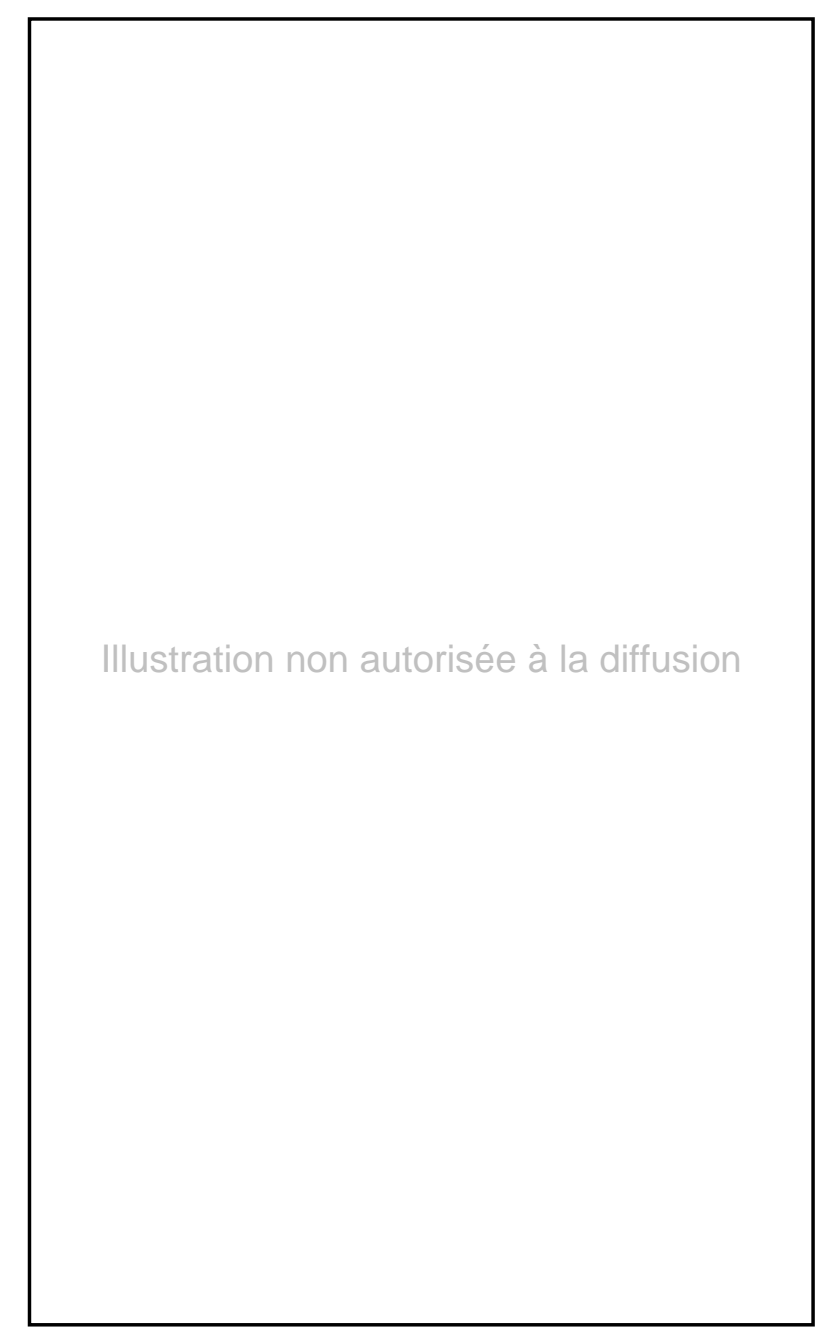

Fig. 28 - Carte de localisation du site des Fourneaux à Vert-SaintDenis (Seine-el-Marne) (dessin L. Costa d'après carte IGN 1/25000).

\section{DES ÉTABLISSEMENTS ANTIQUES AUX TEMPS MÉDIÉVAUX: UNE REMARQUABLE CONTINUITÉ}

La première installation d'importance au lieu-dit Les Fourneaux intervient à La Tène finale (I ${ }^{\text {er }}$ s. avant J.-G.). Dès lors, le site ne cessera d'être occupé jusqu'à la fin du haut Moyen Âge (XI'e s. après J.-C.). L'exploitation du gîte ferrifère ne démarre qu'au VII ${ }^{e}$., au sein d'un habitat rural poursuivant par ailleurs ses activités agropastorales.

L'établissement rural gallo-romain, fondé durant la première moitié $d u I^{\text {er }} \mathrm{s}$. de notre ère, est implanté à $200 \mathrm{~m}$ de la ferme gauloise; il en conserve néanmoins les orientations dominantes, tant dans le tracé de ses enclos et bâtiments que dans celui du parcellaire qui s'est

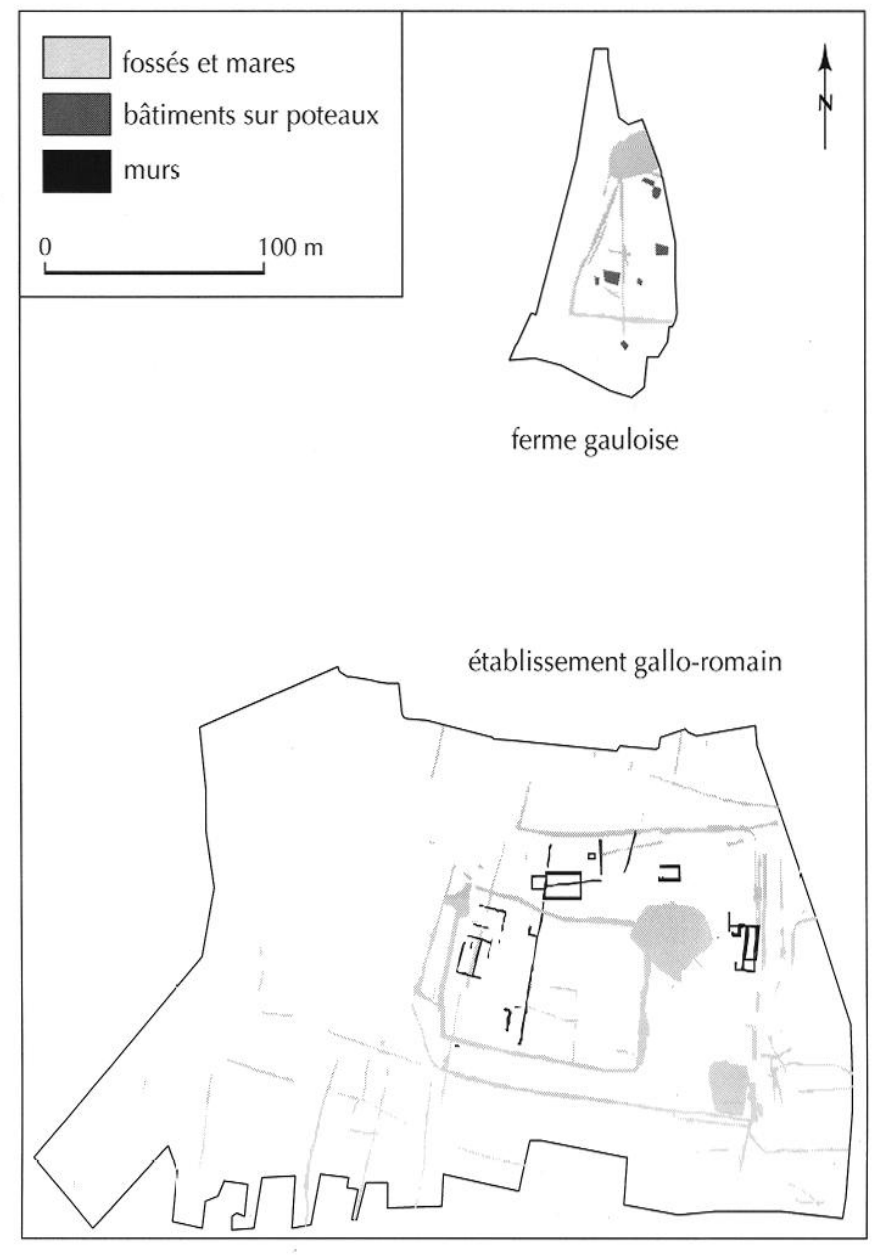

Fig. 29 - Plan masse des installations gauloises et gallo-romaines du site des Fourneaux à Vert-Saint-Denis (Seine-et-Marne). Seules les zones de grande densité de vestiges ont pu être décapées intégralement. Le secteur intermédiaire a été reconnu par le biais de sondages systématiques, non représentés.

développé alentour (fig. 29). Le site connait diverses phases de réaménagement tout au long de la période romaine. Sa physionomie reste cependant relativement stable jusqu'au $V^{c} s$., date à laquelle une nouvelle enceinte est creusée, inscrite à l'intérieur de celle du Haut-Empire. La surface enclose passe alors à 1,5 ha, accusant ainsi une nette restriction. L'occupation du haut Moyen Âge utilisera clairement l'espace remodelé au Bas-Empire.

Au-delà de la seule coïncidence spatiale, la filiation entre les différents habitats est sensible dans leur organisation générale. Les grands axes de structuration de l'espace sont fixés, semble-t-il, dès La Tène finale. L'orientation du système parcellaire restera remarqua- 
blement constante jusqu'à l'établissement du cadastre napoléonien.

L'étude paysagère menée par Sandrine Robert sur le plateau de Sénart met en évidence l'existence d'un réseau souple dans lequel s'intègrent parfaitement les habitats de Vert-Saint-Denis, et auquel participe l'ancienne voie de Melun à Brie-Comte-Robert ${ }^{99}$. La présence de cet axe routier important, à moins de $300 \mathrm{~m}$, a pu jouer un rôle non négligeable dans l'installation et la pérennisation du site, ainsi que dans la diffusion de ses productions.

\section{ÉVOLUTION DE L'HABITAT DU HAUT MOYEN ÂGE}

\section{L'HABITAT MÉROVINGIEN}

Aucun hiatus n'est sensible entre le Bas-Empire et le début de la période mérovingienne. Entre le $\mathrm{IV}^{\mathrm{c}}$ et le $\mathrm{VI}^{\mathrm{e}}$ s., on assiste au passage progressif d'une architecture sur solins et sablières basses à une construction sur poteaux plantés, les bâtiments se succédant aux mêmes emplacements. Parallèlement, de nouvelles structures apparaissent, ainsi, les silos enterrës, les fours domestiques excavés et les fonds de cabane. En dépit de ces transformations technologiques, l'organisation générale du site est largement dépendante des aménagements antérieurs: les vestiges du début de la période mérovingienne sont pour l'essentiel confinés à l'intérieur de l'enceinte creusée au $\mathrm{V}^{\mathrm{e}} \mathrm{s}$., elle-même inscrite dans l'enclos creusé 400 ans auparavant (fig. 30a, $\mathrm{n}^{\circ} 1$ ). L'habitat reste concentré dans la partie occidentale, perpétuant ainsi l'organisation instaurée au $\mathrm{I}^{\mathrm{er}} \mathrm{s}$. Les nouvelles constructions se superposent aux plans précédents, entourées de foyers et de silos enterrés. Quelques aménagements installés autour des bâtiments du HautEmpire, à l'extérieur de l'enclos du $\mathrm{V}^{\mathrm{e}} \mathrm{s}$., témoignent d'une certaine forme de persistance de ces constructions après la période romaine (fig. $30 \mathrm{a}, \mathrm{n}^{\text {os }} 2$ et 3 ).

Par la suite, l'habitat se développe en superficie, gagnant la moitié orientale de l'enclos. À l'ouest du site,

99. S. Robert, Le parcellaire du plateau de Sénart (Seine-et-Marne), in: Chouquer G. (dir.), les formes du paysage -I- Études sur les parcellaires, Préactes du colloque d'Orléans, mars 1996, Paris, éd. Errance, coll. Archéologie aujourd'hui, 1996, p. 11-26.
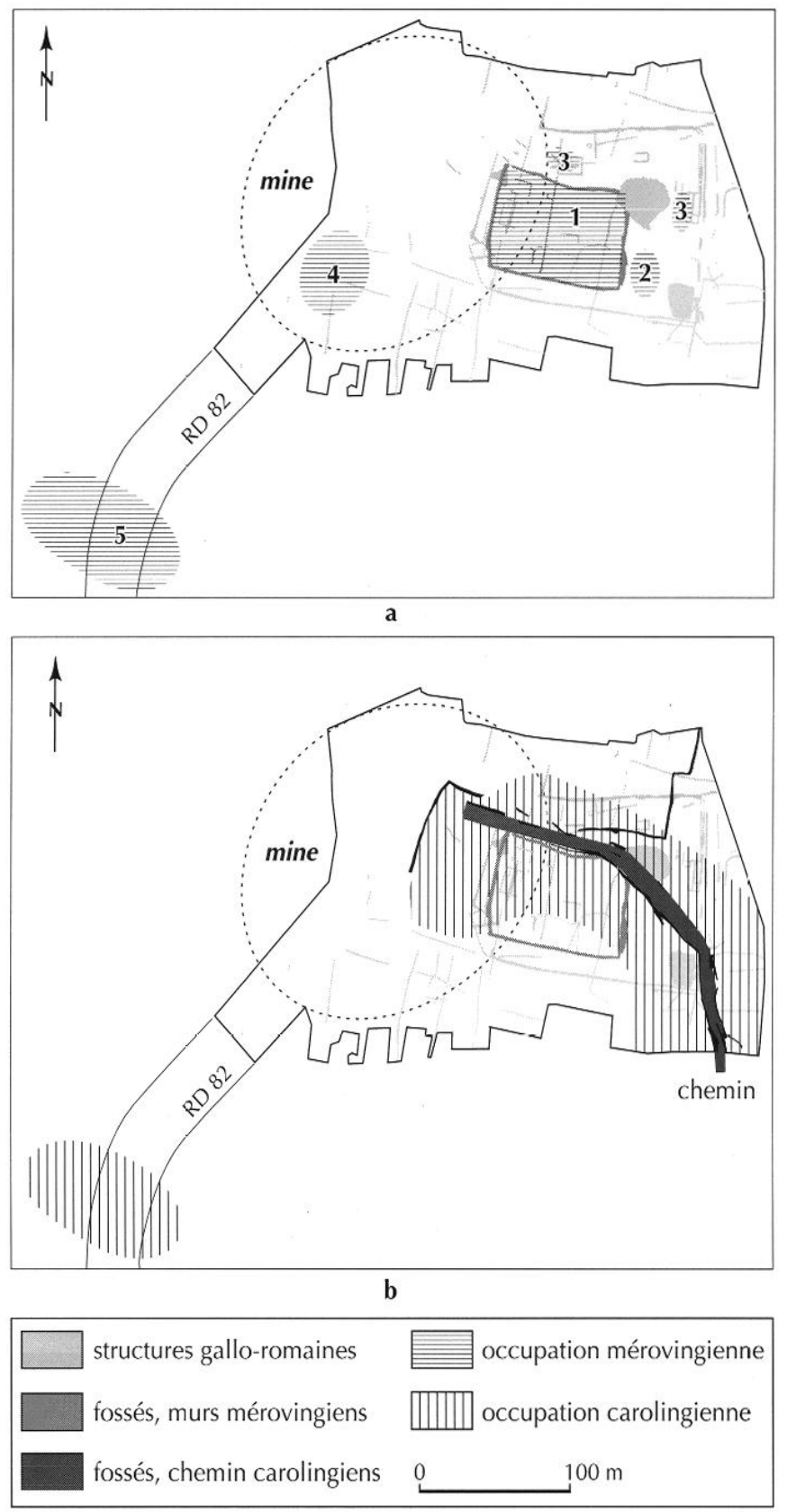

Fig. 30 - L'évolution de l'habitat du haut Moyen Âge du site des Fourneaux à Vert-Saint-Denis (Seine-et-Marne): a, occupation mérovingienne; $b$, occupation carolingienne. Pour chaque période apparaissent les éléments structurant l'espace et les aires d'occupation schématisées (en hachuré). Ies principaux vestiges gallo-romains sont représentés en fond de plan.

de nouvelles installations voient le jour (fig. $30 \mathrm{a}, \mathrm{n}^{\circ} 4$ ). On y retrouve deux emplacements de bâtiments et un grenier sur quatre poteaux, deux fours domestiques et des fosses diverses. Le mobilier céramique trouvé dans ce 
secteur remonte à la fin du $\mathrm{VII}^{\mathrm{e}}$ ou au VIII ${ }^{\mathrm{e}}$ s. La présence de ces unités marginales d'habitation, éloignées jusqu'à plus de $100 \mathrm{~m}$ de l'enclos enserrant l'habitat principal, est peut-être liée à l'activité métallurgique démarrant à la même période.

\section{L'EXPANSION DE L'HABITAT CAROLINGIEN}

Le IX" s. est marqué à Vert-Saint-Denis par un essor de l'habitat. C'est également, semble-t-il, la période de plus grande activité métallurgique. L'occupation transgresse alors les limites précédentes et les structures de cette phase se développent tant vers l'est que vers l'ouest (fig. 30b) .

L'espace est complètement restructuré après cette première phase d'expansion, dans le courant du $\mathrm{X}^{\mathrm{e}} \mathrm{s}$. Un fossé coudé marque alors les nouvelles limites de l'habitat, à l'ouest, empiétant sur un secteur de la mine déjà exploité. L'occupation s'y poursuit jusqu'au $\mathrm{XI}^{\mathrm{e}} \mathrm{s}$. Un chemin permet la desserte de cet enclos. Large d'environ $7 \mathrm{~m}$, il est bordé de fossés qui sont multiples et attestent des réfections. Au sortir du nouvel enclos, le chemin longe l'enceinte du $\mathrm{V}^{\mathrm{e}} \mathrm{s}$., puis oblique vers le sud, esquivant une mare du Haut-Empire. Le fait qu'il évite remarquablement cette mare n'est pas fortuit, elle devait encore constituer un point marquant du paysage. Plusieurs foyers de grillage de minerai y sont d'ailleurs installés, profitant de la dépression toujours sensible pour s'abriter du vent, ou d'un espace plus libre au sein de l'habitat. L'angle ainsi formé par le chemin est corrigé dans un second temps, un nouveau fossé bordier traversant la mare en diagonale, selon un tracé plus rectiligne.

La présence de cette voie de desserte, par les facilités de transport de matériaux qu'elle apporte, a sans doute conditionné l'installation des deux bas fourneaux les plus éloignés de la zone d'extraction (5739) au sud-est et un probable bas fourneau disparu, dont l'emplacement est signalé par des dépotoirs à scories au niveau de l'angle du chemin.

Ce chemin servira d'épine dorsale autour de laquelle l'habitat va se restructurer. Des bâtiments, silos, fours domestiques, fonds de cabane, etc. s'installent de part et d'autre, dans des espaces cloisonnés par des fossés. La physionomie du site s'en trouve modifiée, passant d'un habitat nucléaire à une structure de "village-rue ", désormais totalement affranchie du cadre fixé par l'enceinte gallo-romaine. La présence de structures tardives (fin $\mathrm{XI}^{e}$ s.) le long du chemin à l'est tend à démontrer une progression de l'habitat dans cette direction.

\section{STATUT DE L'HABITAT DU HAUT MOYEN ÂGE}

L'île-de-France apparait comme une région phare en ce qui concerne la connaissance des habitats ruraux du haut Moyen Âge. Ce thème de recherche, initié dès la fin des années 1970 par les travaux de chercheurs tels que Rémy Guadagnin sur le Pays-de-France (95), a ensuite été nourri par le développement de l'archéologie préventive. En 1992, à l'occasion de l'exposition "L'Île-deFrance de Clovis à Hugues Capet », Jean Chapelot recensait plus de quarante sites de ce type fouillés dans la région ${ }^{100}$. Sur le seul plateau briard, le suivi archéologique d'opérations de grands travaux tels que Marnela-Vallée, l'autoroute A5 et le TGV Nord a permis la fouille sur plusieurs hectares d'une douzaine d'habitats ruraux du haut Moyen Âge. L'étude du site des Fourneaux s'inscrit donc dans un contexte bien documenté, offrant des possibilités de confrontation.

Le site des Fourneaux est occupé sans discontinuité apparente depuis le Bas-Empire jusqu'à la fin du XI' s. Aucun hiatus n'a été perçu dans le spectre mobilier entre ces deux dates. Six siècles d'aménagements successifs du haut Moyen Âge conduisent à relativiser l'importance de l'habitat. Son plan de masse résulte de la surimpression des vestiges pendant toute cette longue période et n'est représentatif ni de son étendue ni de sa densité à un instant donné.

Dans sa phase de plus grande extension (IX ${ }^{\mathrm{c}}-\mathrm{X}^{\mathrm{c}} \mathrm{s}$.), l'habitat du haut Moyen Âge couvre 3 ha du décapage de Vert-Saint-Denis. Si le site n'est pas totalement délimité, les vestiges se poursuivant vraisemblablement au sud-est en dehors de notre emprise, le résultat négatif des prospections de surface entreprises dans les parcelles limitrophes laisse supposer que l'on se trouve ici sur les marges de l'habitat.

En comparaison des sites contemporains fouillés dans la région, celui des Fourneaux peut être classé, par sa surface, parmi les habitats de taille moyenne, à l'instar de celui tout proche du Climat des Terres Noires à Saint-

100. J. Chapelot, L'habitat rural: organisation et nature, in: l'île-deFrance de Clovis à Hugues Capet du V' siècle au X' siècle, Catalogue de l'exposition du musée archéologique départemental du Val-d'Oise, Service régional de l'archéologie d'île-de-France, éd. du Valhermeil, 1993, p. 178-199. 
Germain-Laxis. La densité des vestiges est telle que l'on ne peut préciser, pour chaque phase, le nombre d'unités d'exploitation qui le composent. Un autre indice tangible dans l'approche de la hiérarchie des établissements repose sur la présence d'un cimetière ou d'un lieu de culte, témoignant du pouvoir attractif d'un habitat, voire d'une présence ecclésiastique. Malgré sa remarquable longévité, le site des Fourneaux en est dépourvu. Vingt et une sépultures sont attribuables à cette période. Regroupées en petites zones d'inhumation ou isolées, parfois reléguées dans le comblement de fosses d'extraction de minerai, elles soulèvent le problème du statut de ces quelques individus exclus des cimetières, mais ne constituent certes pas l'ensemble de la population pendant les six siècles d'occupation.

En dehors des aménagements directement liés à la métallurgie, le site rassemble les structures habituellement rencontrées sur les habitats ruraux à vocation agricole: bâtiments et greniers sur poteaux, silos, fonds de cabane, fours domestiques, puits à eau... Outre les structures destinées à la conservation des récoltes, les paléosemences et l'outillage (faucilles) témoignent de la pratique de l'agriculture. La part de l'élevage ne peut encore être appréciée, car l'étude des restes fauniques, retrouvés en abondance, n'a pas encore été faite, fautc dc crédits. La densité et la variété des dépotoirs osseux, associées à la découverte de couteaux et pierres à aiguiser, permettent de définir deux secteurs plus spécifiquement voués aux travaux de boucherie. Le premier est daté de la fin du VIII' ou du début du IX' ${ }^{c}$ s., le second remonte au $\mathrm{X}^{\mathrm{e}} \mathrm{s}$. Tous deux sont contemporains de l'activité métallurgique; ils témoignent d'une sectorisation, peut-être d'une spécialisation, des activités au sein de l'habitat.

Comme sur la plupart des sites contemporains, le tissage est attesté par la présence de pesons, navettes ou broches de tisserands. Plusieurs fonds de cabane présentaient sur leur sol les empreintes de montants pouvant appartenir à un métier à tisser vertical. Des poinçons en fer pourraient se rapporter au corroyage.

Hormis l'importance des structures liées à la métallurgie, le site des Fourneaux ne se démarque en rien des habitats ruraux contemporains. Les activités agricoles et domestiques habituelles y sont pratiquées, tandis que l'absence de cimetière nous conduit à le qualifier de « hameau " plutôt que de « village ".

Il convient de signaler la présence d'autres zones d'occupation du haut Moyen Âge sur les versants sud et ouest de la butte stampienne, au lieu-dit Les Hautes Billes. L'évaluation archéologique effectuée sur la suite du tracé de la déviation de la RD 82 vient prolonger le décapage en direction du sud-ouest (fig. $30, n^{\circ} 5$ ). Un secteur dense en structures y a été mis au jour, à $200 \mathrm{~m}$ du cœur du site des Fourneaux ${ }^{101}$. L'occupation est datée du VIII' au X $\mathrm{X}^{\mathrm{e}} \mathrm{s}$. Elle rassemble des vestiges d'habitat (trous de poteaux, fours domestiques, fosses diverses), mais également un bas fourneau du même type que ceux qui ont été découverts aux Fourneaux, et qui n'en est éloigné que d'une centaine de mètres. La faible largeur de la zone décapée lors de l'évaluation $(8 \mathrm{~m})$ ne permet pas d'apprécier l'importance et l'extension de ce nouvel habitat. À environ $100 \mathrm{~m}$ à l'ouest de ce gisement, d'autres aménagements du haut Moyen Âge implantés sur des vestiges gallo-romains avaient été révélés en 1993 lors de sondages dans le cadre de l'opération archéologique sur la ville nouvelle de Sénart. Là encore, des scories ont été retrouvées dans le remplissage des structures.

Le hameau des Fourneaux s'inscrit donc dans un tissu extrêmement dense d'occupation. Si cette structure polynucléaire n'est pas exceptionnelle (elle a déjà été rencontrée sur des sites carolingiens étudiés sur de grandes surfaccs), cllc est peut-être ici induite par la présence de la mine. Les différents noyaux d'habitation contemporains ont en effet manifestement participé à l'exploitation du minerai de fer. Les liens qui les unissent, leur éventuelle hiérarchisation, ne peuvent être définis en l'état de la documentation. Il faudra également attendre de nouvelles interventions pour valider le schéma d'une multiplication d'installations dispersées autour de la mine dont elles se partageraient l'exploitation.

\section{LE COMPLEXE MÉTALLURGIQUE ET MINIER}

\section{CHRONOLOGIE DE L'ACTIVITÉ MINIÈRE ET MÉTALLURGIQUE}

En dépit de la profusion de vestiges liés à la métallurgie (fig. 31), le calage chronologique de la fin de cette activité reste un problème délicat.

101. I'évaluation sur la RD 82 a été réalisée en 1994, tandis que se déroulait le sauvetage des Fourneaux, par Anne-Aimée Lichon (AFAN). 


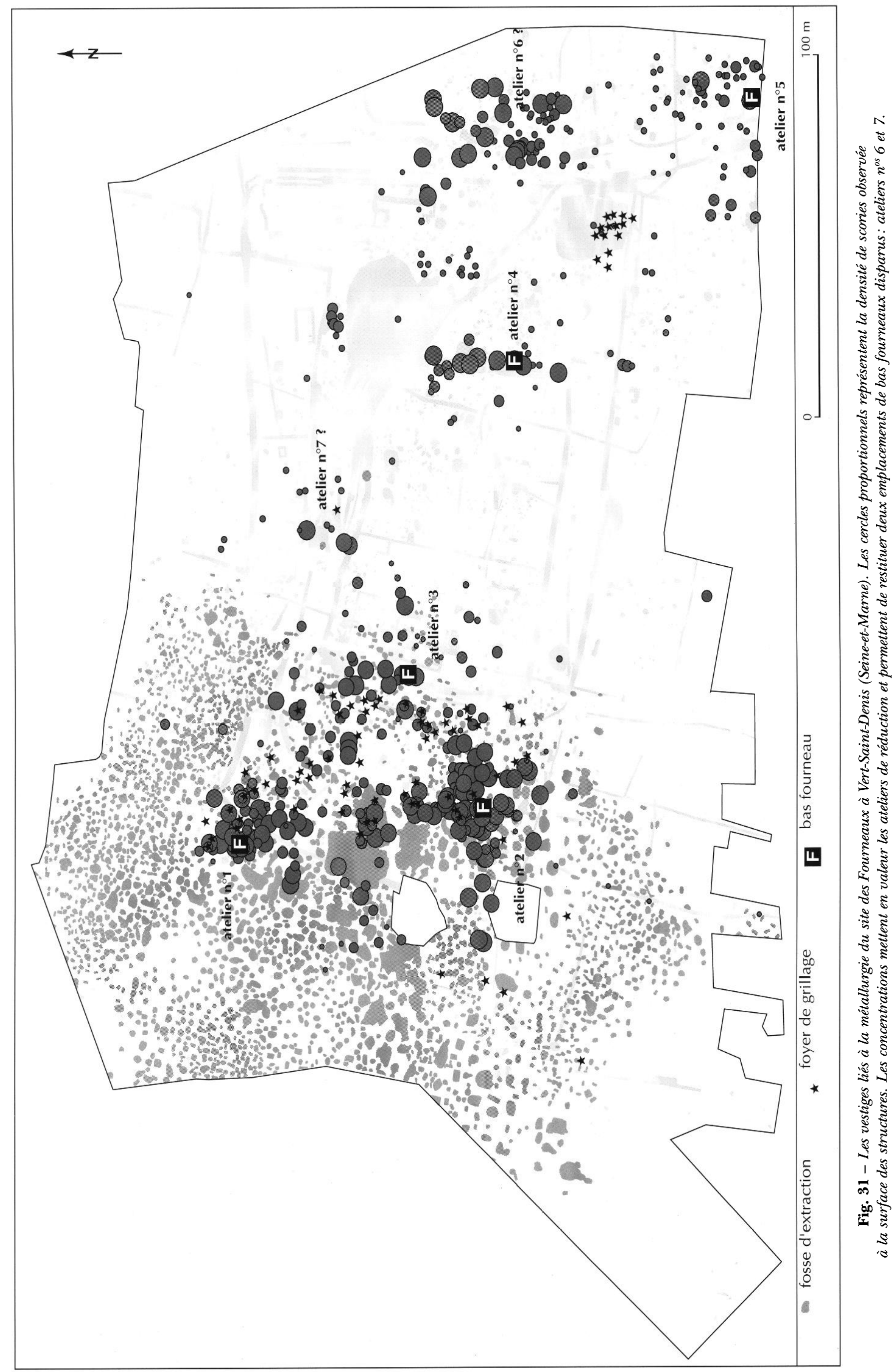


Les éléments appuyant une datation absolue sont rares tant le mobilier archéologique contenu dans ces structures est indigent. Sur les cinq bas fourneaux, un seul (10020) a livré de la céramique : 3 tessons de poterie granuleuse, attribuables sans plus de précision au haut Moyen Âge. La fouille des foyers de grillage ne s'est pas avérée beaucoup plus fructueuse. Quant aux fosses d'extraction, étant donné leur mode de remplissage - comblement rapide à l'aide des stériles provenant des puits voisins - leur mobilier est rare et peu représentatif. Il provient en effet du sédiment mobilisé plutôt que de rejets contemporains du creusement, et a toute chance d'être résiduel. Ainsi, les ensembles les plus abondants sont datés du I Irr s. après J.-C. ; ils sont localisés dans le secteur d'occupation gallo-romaine où les puits traversent des niveaux riches en céramique. Le mobilier récolté sur la mine doit donc être considéré avec précaution, il ne peut nous fournir qu'un terminus post quem. Quatre-vingts fosses d'extraction ont livré du mobilier couvrant la fin de la période mérovingienne et la période carolingienne. On peut souligner la présence de deux fibules ansées symétriques datables du VII s. à proximité du bas fourneau 3001 (atelier $n^{\circ}$ 1) ${ }^{102}$. Le comblement charbonneux de trois structures liées à la métallurgie a pu être daté par radiocarbone ${ }^{103}$. Les dates s'échelonnent entre le début du VIII ${ }^{\mathrm{c}}$ et le $\mathrm{X}^{\mathrm{e}} \mathrm{s}$.

Les éléments nous manquent aussi pour fixer précisément le démarrage de l'activité. L'apparition de déchets dans les structures d'habitat bien datées par leur mobilier intervient au VIII's. Leur permanence dans les remplissages à partir de cette date ne peut être considérée comme un critère fiable du maintien de l'activité, tant le sol devait être jonché de scories. Le jalon le plus récent est constitué par un fond de cabane daté de la fin du $\mathrm{X}^{\mathrm{e}}$ ou du XI's., et recoupé par une fosse d'extraction. Il atteste la poursuite de l'exploitation du minerai jusqu'à cette date.

En corrélant ces indices, on peut situer le début de l'activité métallurgique à la fin $\mathrm{du} \mathrm{VII}^{\mathrm{e}}$ ou au début du VIII $^{\mathrm{e}}$ s., les dernières fosses d'extraction étant datées du $\mathrm{XI}^{\mathrm{e}} \mathrm{s}$. Son terme coïnciderait ainsi avec la fin de l'occupation du site.

Le maintien de l'activité tout au long de cette période est loin d'être assuré, les données chronologiques sont

102. Renseignement de Bruno Foucray (SDA).

103. Datations réalisées par le Centre de datation par le radiocarbone (Lyon), échantillons I.y-6784, Ly-6786 et Ly-6787. trop ténues pour que l'on puisse apprécier dans le détail la continuité ou les clivages de l'exploitation. De même, la durée de fonctionnement et la chronologie relative des différents ateliers ne peuvent être précisées. L'obtention de nouvelles datations par radiocarbone nous serait ici extrêmement précieuse.

\section{L'EXTRACTION DU MINERAI DE FER}

Plus de 2500 puits d'extraction du minerai de fer ont été dénombrés sur les 2 ha de mine décapés. Si l'extraction en contexte karstique est connue dans d'autres régions (Mangin et al., 1992), la documentation sur les mines en plateau limoneux est encore peu abondante en Europe, en l'absence de fouille.

Les conditions d'urgence dans lesquelles s'est déroulé le sauvetage du site des Fourneaux ont largement influencé le choix des méthodes de fouille. Une grande partie du secteur occupé par la mine (1,5 ha) a dû en effet être livré aux aménageurs moins de quinze jours après la fin de son décapage. Seule une dizaine de puits a été fouillée à la main. Le choix de cet échantillon s'est porté sur les fosses proches des bas fourneaux, dans lesquelles ont été rejetés les déchets de rćduction. En dehors de ces dépotoirs, le remplissage des puits est stérile. Une fouille manuelle d'un plus grand échantillon se trouvait irréalisable dans les délais impartis. Les moyens mécaniques ont donc été largement utilisés.

De grandes tranchées traversent la mine de part en part dans le sens de la pente (sud-ouest/nord-est) et selon l'axe perpendiculaire. Elles répondent à plusieurs objectifs: d'une part, l'observation des niveaux géologiques et le suivi du banc de minerai; d'autre part, la coupe de nombreuses fosses d'extraction et l'approche de leur typologie, mode de remplissage et relations stratigraphiques; enfin, du minerai a été prélevé en divers points du site, afin de fournir des échantillons pour analyses et d'apporter des données quantitatives sur la productivité de l'extraction.

Ces tranchées ont été complétées par des sondages ponctuels, dispersés sur la surface, afin de vérifier la profondeur du minerai et la morphologie des fosses.

Deux secteurs $\left(1340 \mathrm{~m}^{2}\right)$ ont été décaissés sur près de $1 \mathrm{~m}$ de profondeur. Le limon superficiel, fortement perturbé dans la zone centrale, rendait difficile la lecture 


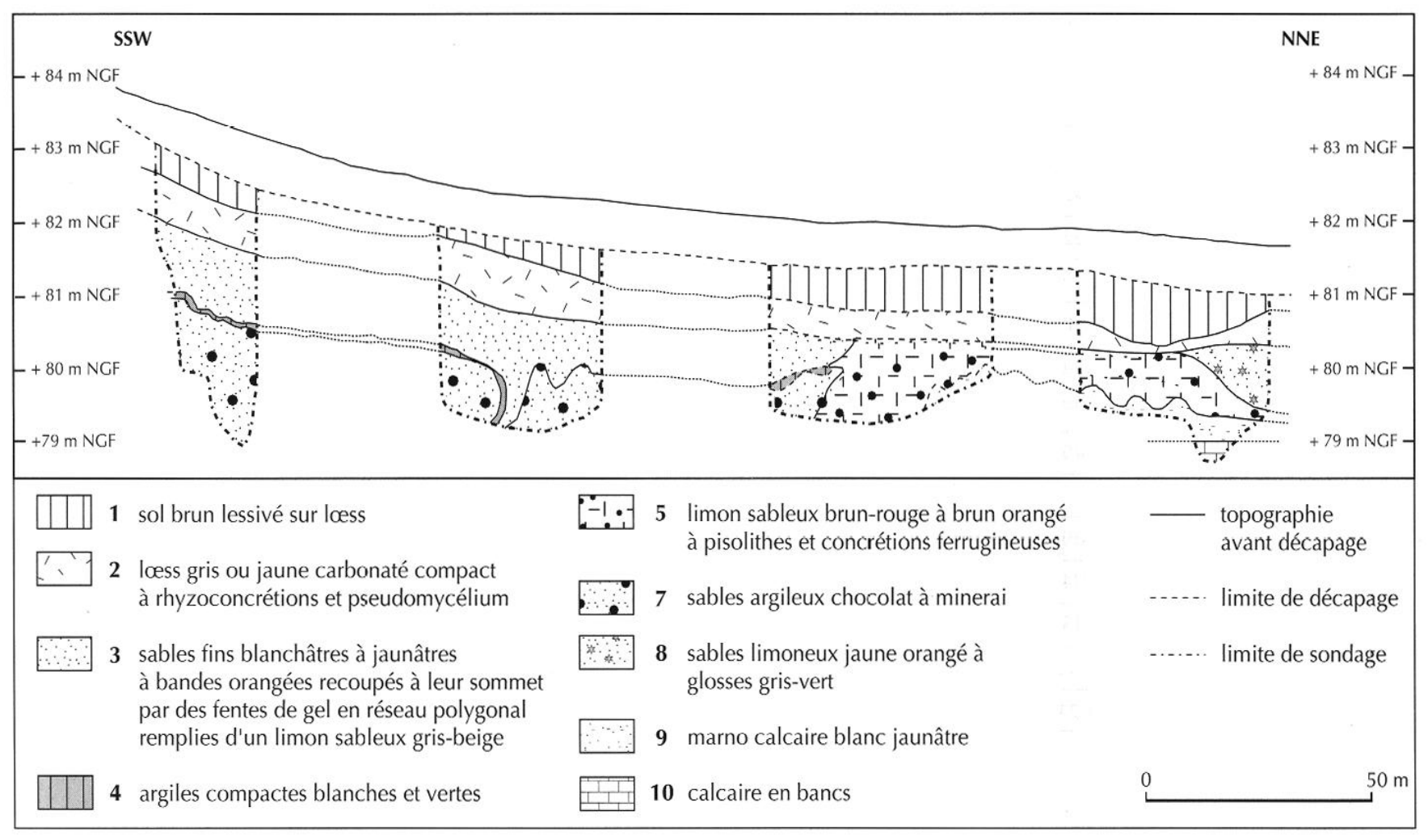

Fig. 32 - Coupe synthétique des formations géologiques du site des Fourneaux à Vert-Saint-Denis (Seine-et-Marne). La couche de cailloutis de silex gélifractés (6) ne peut pas être représentée à l'échelle utilisée.

des fosses, le décapage jusqu'au niveau du sable a permis d'atteindre un degré de perception satisfaisant, afin de compléter les informations de formes et de densité, et d'évaluer la validité des observations recueillies en surface sur ces secteurs difficiles.

\section{CARACTÈre DU GITTE FERRIFÈre DE VERT-SAINT-DENIS}

Les formations recherchées par les anciens métallurgistes sont de deux sortes (fig. 32) : d'une part, le limon sableux chocolat à pisolithes et rognons de minerai de fer observé sous le sable en sommet de butte et, d'autre part, le limon sableux brun-rouge à brun orangé à pisolithes et concrétions ferrugineuses apparaissant sous le loess en bas de butte. Ce dernier niveau correspond vraisemblablement au remaniement très pédogénisé des limons chocolat observés en sommet de butte. Bien que beaucoup moins riche en minerai que la formation chocolat, cette unité stratigraphique présente l'avantage d'être moins profonde et donc plus facile à atteindre pour les mineurs.
Des échantillons de minerai ferrugineux provenant de divers endroits de la zone d'extraction ont été analysés au Centre de recherches pétrographiques et géochimiques (CRPG) de Vandouvre-lès-Nancy, sous la responsabilité de A. Ploquin (Ploquin, 1994a; Fluzin et al., infra, p. 101-121). Les valeurs obtenues sont homogènes (tabl. II). Les teneurs en $\mathrm{Fe}_{2} \mathrm{O}_{3}$ total varient de $75,53 \%$ à $80,56 \%$, celles en silice et en alumine sont comprises respectivement entre $1,98 \%$ et $6,07 \%$, et entre $0,41 \%$ et $1,69 \%$. La teneur en eau est de l'ordre de $15 \%$. Cette homogénéité des résultats indique bien que le minerai provenant des limons chocolat (échantillons 1676 à 1680 et 1682) et celui issu des limons sableux brun orangé (échantillons 1665 à 1667 ) ont la même origine.

Le très faible pourcentage de silice, d'alumine, de calcium, de même que la concentration très remarquable en oxydes de fer sont les traits marquants de ce minerai. Comme on pouvait s'y attendre, les parties les plus dures (ou noyaux) sont très fortement concentrées en oxydes $(81,44 \%)$, avec pour corollaire une infime teneur en silice $(1,98 \%), c f$. échantillon 1676 . Ces caractères sont 
Tabl. II - Résultats de l'analyse chimique par émission ICP spectroscopie de minerais et de scories de réduction (A. Ploquin, CRPG de Vandeuvre-lès-Nancy).

MINERAIS

\begin{tabular}{|c|c|c|c|c|c|c|c|c|c|}
\hline \multicolumn{10}{|c|}{ Échantillons } \\
\hline Éléments (\%) & $\mathbf{1 6 6 5}$ & $\mathbf{1 6 6 6}$ & $\mathbf{1 6 6 7}$ & $\mathbf{1 6 7 6}$ & $\mathbf{1 6 7 7}$ & $\mathbf{1 6 7 8}$ & $\mathbf{1 6 7 9}$ & $\mathbf{1 6 8 0}$ & $\mathbf{1 6 8 2}$ \\
\hline $\mathrm{SIO}_{2}$ & 5,22 & 3,53 & 4,28 & 2,24 & 1,98 & 3,56 & 6,07 & 3,18 & 5,68 \\
\hline $\mathrm{Al}_{2} \mathrm{O}_{3}$ & 1,15 & 0,99 & 1,08 & 0,42 & 0,41 & 0,99 & 1,56 & 0,95 & 1,69 \\
\hline $\mathrm{Fe}_{2} \mathrm{O}_{3}$ & 76,39 & 79,14 & 76,71 & 80,56 & 81,44 & 77,68 & 75,53 & 80,14 & 77,48 \\
\hline $\mathrm{MnO}$ & 0,24 & 0,23 & 0,47 & 0,34 & 0,27 & 0,38 & 0,28 & 0,21 & 0,32 \\
\hline $\mathrm{MgO}$ & 0,27 & 0,24 & 0,27 & 0,17 & 0,14 & 0,24 & 0,23 & 0,15 & 0,20 \\
\hline $\mathrm{CaO}$ & 0,45 & 0,23 & 0,29 & 0,13 & 0,06 & 0,20 & 0,20 & - & 0,10 \\
\hline $\mathrm{Na}_{2} \mathrm{O}$ & - & - & - & - & - & - & - & - & - \\
\hline $\mathrm{K}_{2} \mathrm{O}$ & 0,09 & 0,14 & 0,14 & 0,02 & 0,06 & 0,06 & 0,11 & 0,05 & 0,18 \\
\hline $\mathrm{TIO}_{2}$ & 0,04 & 0,02 & 0,03 & 0,02 & 0,02 & 0,03 & 0,06 & 0,03 & 0,07 \\
\hline $\mathrm{P}_{2} \mathrm{O}_{5}$ & 0,45 & 0,35 & 0,47 & 0,62 & 0,51 & 0,80 & 0,74 & 0,36 & 0,43 \\
\hline $\mathrm{Perte}$ au feu & 15,41 & 15,56 & 16,30 & 15,29 & 14,78 & 15,91 & 15,31 & 14,28 & 13,99 \\
\hline $\mathrm{Total}$ & $\mathbf{9 9 , 7 1}$ & $\mathbf{1 0 0 , 4 3}$ & $\mathbf{1 0 0 , 0 4}$ & $\mathbf{9 9 , 8 1}$ & $\mathbf{9 9 , 6 7}$ & $\mathbf{9 9 , 8 5}$ & $\mathbf{1 0 0 , 0 9}$ & $\mathbf{9 9 , 3 5}$ & $\mathbf{1 0 0 , 1 4}$ \\
\hline
\end{tabular}

SCORIES

\begin{tabular}{|c|c|c|c|c|c|c|c|c|c|c|c|}
\hline \multicolumn{10}{|c|}{ Échantillons } \\
\hline Éléments (\%) & $\mathbf{1 6 6 8}$ & $\mathbf{1 6 6 9}$ & $\mathbf{1 6 7 0}$ & $\mathbf{1 6 7 1}$ & $\mathbf{1 6 7 2}$ & $\mathbf{1 6 7 3}$ & $\mathbf{1 6 7 4}$ & $\mathbf{1 6 8 1}$ & $\mathbf{1 6 8 3}$ & $\mathbf{1 6 8 5}$ & $\mathbf{1 6 8 6}$ \\
\hline $\mathrm{SIO}_{2}$ & 24,84 & 20,44 & 23,71 & 26,11 & 30,25 & 26,57 & 26,95 & 25,43 & 28,33 & 25,43 & 25,02 \\
\hline $\mathrm{Al}_{2} \mathrm{O}_{3}$ & 4,13 & 3,01 & 3,63 & 4,35 & 5,42 & 4,75 & 4,02 & 3,19 & 4,60 & 3,73 & 3,96 \\
\hline $\mathrm{Fe}_{2} \mathrm{O}_{3}$ & 65,97 & 75,02 & 75,18 & 71,56 & 63,46 & 63,45 & 66,15 & 74,15 & 67,02 & 63,43 & 67,85 \\
\hline $\mathrm{MnO}$ & 1,01 & 0,74 & 1,04 & 0,48 & 1,08 & 1,75 & 0,95 & 0,92 & 1,35 & 0,54 & 0,89 \\
\hline $\mathrm{MgO}$ & 0,48 & 0,41 & 0,37 & 0,48 & 0,51 & 0,60 & 0,49 & 0,37 & 0,59 & 0,37 & 0,41 \\
\hline $\mathrm{CaO}$ & 1,27 & 0,90 & 1,24 & 1,96 & 2,29 & 5,42 & 2,37 & 0,91 & 1,50 & 1,16 & 0,82 \\
\hline $\mathrm{Na}_{2} \mathrm{O}$ & 0,02 & - & - & - & - & 0,05 & - & - & 0,06 & - & 0,05 \\
\hline $\mathrm{K}_{2} \mathrm{O}$ & 0,63 & 0,38 & 0,65 & 0,69 & 1,11 & 0,98 & 0,81 & 0,66 & 0,88 & 0,55 & 0,74 \\
\hline $\mathrm{TIO}_{2}$ & 0,22 & 0,16 & 0,14 & 0,18 & 0,20 & 0,21 & 0,16 & 0,17 & 0,22 & 0,16 & 0,20 \\
\hline $\mathrm{P}_{2} \mathrm{O}_{5}$ & 0,82 & 0,96 & 0,47 & 0,46 & 0,45 & 0,52 & 0,50 & 0,93 & 0,61 & 0,50 & 0,62 \\
\hline $\mathrm{Perte}_{\mathrm{au}}$ feu & 0,59 & $-1,99$ & $-6,78$ & $-6,40$ & $-4,80$ & $-5,39$ & $-2,20$ & $-6,88$ & $-5,20$ & 4,10 & $-1,20$ \\
\hline $\mathrm{Total}$ & $\mathbf{9 9 , 9 8}$ & $\mathbf{1 0 0 , 0 3}$ & $\mathbf{9 9 , 6 5}$ & $\mathbf{9 9 , 8 7}$ & $\mathbf{9 9 , 9 7}$ & $\mathbf{9 8 , 9 1}$ & $\mathbf{1 0 0 , 2 0}$ & $\mathbf{9 9 , 8 4}$ & $\mathbf{9 9 , 9 6}$ & $\mathbf{9 9 , 9 8}$ & $\mathbf{9 9 , 3 6}$ \\
\hline
\end{tabular}

en opposition complète avec l'idée généralement admise selon laquelle ces minerais de plateau sont siliceux et très peu concentrés. À l'inverse, l'enveloppe des rognons de minerai contient un peu plus de silice. À côté des rognons concentrés, on rencontre des nodules de petite taille, un peu moins concentrés en oxydes. Ces différences de composition restent cependant minimes et on peut exclure qu'elles aient eu une incidence dans la sélection des métallurgistes. D'un point de vue théorique, ces minerais sont peu fondants, dans la mesure où ils sont pauvres en silice. Ils sont donc difficiles à réduire bien que très riches en oxydes.

Des analyses minéralogiques par diffraction $\mathrm{X}$ ont été réalisées sur un échantillon de minerai par Philippe Ildefonse du Laboratoire de minéralogie-cristallographie de Jussieu (laboratoire mixte Paris VI-Paris VII). Cette 
analyse a montré que le minerai est principalement constitué de gœthite bien cristallisée, associée à de la lépidocrocite et à du quartz en très faible proportion, sans qu'il y ait trace de minéraux alumineux. L'association gœthite-lépidocrocite est intéressante, car elle implique des conditions physico-chimiques très particulières au moment de la formation du minerai, en particulier au niveau des valeurs du potentiel redox $\left(E_{h}\right)$ et de la température.

En ce qui concerne l'origine géologique du minerai, les différents spécialistes consultés ont émis deux types d'hypothèses. Daniel Obert (Laboratoire de géotectonique, Université Paris VI) et Yvette Dewolf (Institut Buffon, Université Paris VII) penchent pour une origine ancienne et attribuent ce minerai au remaniement d'un sol de type latéritique tertiaire, avec accumulation au niveau de dépressions karstiques. Cette hypothèse a été appuyée par Nicolas Fédoroff (INRA de Grignon), qui souligne la ressemblance entre ce minerai et celui provenant des formations latéritiques de l'ouest africain. Par contre, Médard Thiry (École des mines de Fontainebleau) propose une version totalement différente pour expliquer la genèse de ces hydroxydes. Il pense en effet que le minerai résulte du lessivage quaternaire des minéraux ferromagnésiens initialcment contcnus dans les sables de Fontainebleau. Le fer ainsi dissous passe dans les nappes phréatiques et est piégé au contact du calcaire, par augmentation du $\mathrm{pH}$.

\section{LES TECHNIQUES D'EXTRACTION}

La méthode d'extraction utilisée à Vert-Saint-Denis consiste en l'ouverture de puits traversant successivement les niveaux de limons et de sables jusqu'à atteindre le banc de minerai (fig. 33 et 34). Les parois sont verticales, mais des affouillements limités destinés à dégager quelques rognons de minerai sont parfois visibles à la base des fosses. L'instabilité du terrain rend cependant périlleux tout travail en sape et le banc n'a jamais été exploité dans des fosses en cloche ou avec galeries souterraines. Les limons et sables encaissants n'ont pas conservé de trace d'outil.

La collecte du minerai suscite quelques interrogations: y avait-il une sélection, un tri, et selon quels critères? Peut-on envisager un tamisage? La présence, au fond des fosses, d'une couche d'argile à pisolithes provenant du banc de minerai remanié nous incite à

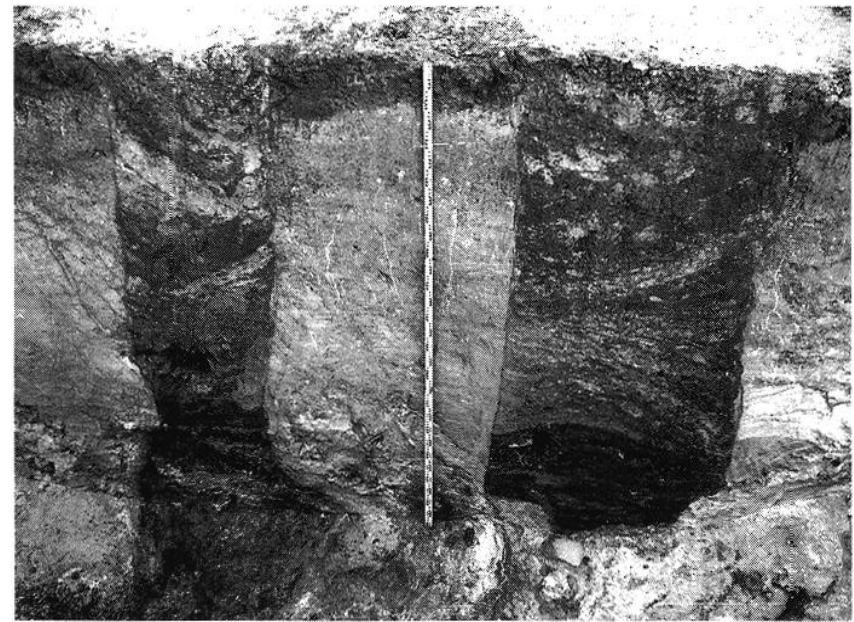

Fig. 33 - Les Fourneaux à Vert-Saint-Denis (Seine-et-Marne): vue en coupe de deux puits d'extraction. La mire mesure 3 m (cliché AFAN).

envisager l'extraction des rognons palpables à la main et à exclure l'hypothèse d'un prélèvement en masse du sédiment suivi d'un tamisage. Un piochon, retrouvé dans un puits à eau, a pu servir à dégager les rognons de leur gangue argileuse.

La taille des puits est variable: leur profondeur dépend évidemment du niveau auquel se trouve le minerai, mais leurs dimensions en plan sont très disparates. Quatre groupes de fosses ont été définis en fonction de leur superficie (fig. 35). Les deux groupes extrêmes témoignent de modes d'extraction sensiblement différents (groupes I et IV décrits ci-après).

Le groupe I rassemble le plus grand nombre de fosses (1266). Leur superficie est inférieure à $1,5 \mathrm{~m}^{2}$. Leur forme est en général très régulière, souvent parfaitement circulaire ou rectangulaire. Les dimensions les plus fréquentes pour ces dernières sont comprises entre $1,10 \mathrm{~m}$ et $1,60 \mathrm{~m}$ pour la longueur et $0,60 \mathrm{~m}$ à $1 \mathrm{~m}$ en largeur correspondante. En certains points du site, la régularité de ces fosses est frappante; elle évoque un gabarit dicté sans doute par l'amplitude du geste des mineurs. Ces puits sont des ouvrages individuels: un seul mineur suffit à les creuser, assisté d'une personne en surface pour l'évacuation des déblais. Aucun indice de blindage des parois n'a été décelé, le temps nécessaire à l'ouverture d'un puits n'excède vraisemblablement pas un jour ou deux; les risques d'effondrement sont par conséquent réduits. L'absence d'érosion des bords indique un comblement rapide. 


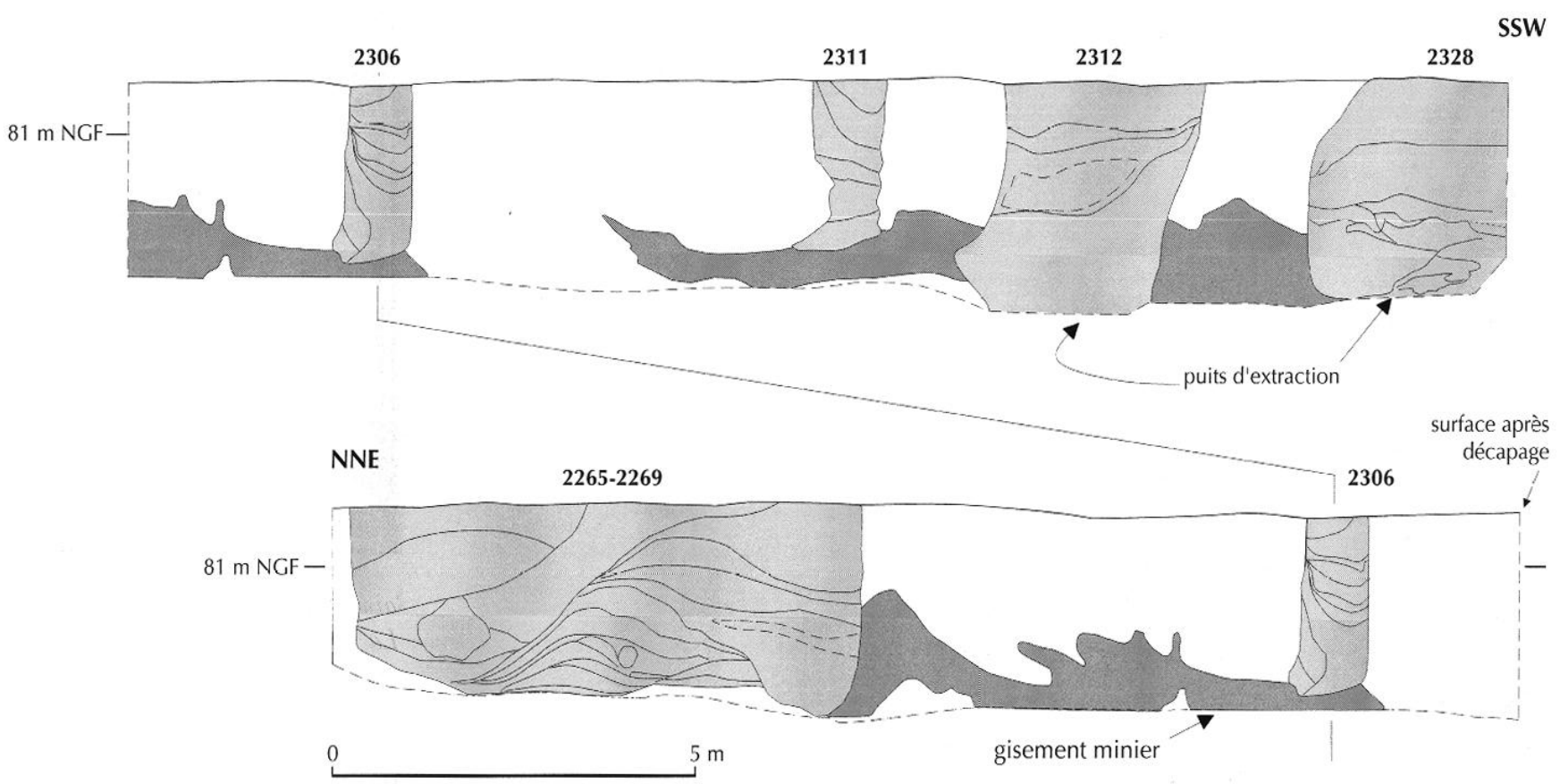

Fig. 34 - Les Fourneaux à Vert-Saint-Denis (Seine-et-Marne): relevé en coupe d'une des tranchées creusées à la pelle mécanique. Le banc de minerai apparaît en gris sombre (relevé J.J. Bahain et L. Costa, 1994; dessin V. Gouslard).

Des séries de fosses regroupées en chapelets apparaissent sur le plan de masse, principalement dans le secteur nord. Elles rassemblent 4 ou 5 structures de même gabarit, disposées à peu de distance, alignées ou rayonnantes autour d'un petit espace vide. Cette organisation évoque des puits creusés successivement par un même individu ou simultanément par plusieurs mineurs utilisant un même tas de déblais, au centre. Dans l'une des tranchées ouvertes à la pelle mécanique, deux fosses voisines ont le même module, et exactement la même profondeur. L'une d'elles n'atteint pas le banc de minerai, plongeant verticalement à cet endroit. Ces deux fosses semblent bien avoir été creusées en même temps, l'une d'elles a été abandonnée devant l'absence de minerai. L'association de ces ensembles avec l'extraction nécessaire à une fournée de réduction est tentante. Cette hypothèse devra cependant être étayée par l'évaluation des quantités de minerai extraites et de celles requises par le processus de réduction en fonction des déchets retrouvés.

Le groupe IV rassemble des structures de forme tentaculaire, aux limites difficilement perceptibles et pouvant couvrir de grandes surfaces (jusqu'à $240 \mathrm{~m}^{2}$ ). Elles pcuvcnt résulter du recoupement de multiples fosses non individualisables en plan. Cependant de vastes creusements ont été observés en coupe. Leur taille, leur morphologie et leur stratigraphie sont moins stéréotypées que celles des fosses du groupe I. On peut en déduire différents procédés de mise en œuvre.

- F2269 (fig. 34) : la fosse mesure $7 \mathrm{~m}$ de large dans l'axe de la coupe. Son bord méridional est vertical et il est plus sinueux au nord. Le fond est irrégulier; il dessine une cuvette étroite au nord, remonte brutalement, puis redevient concave. Le comblement est constitué d'une succession de lits de matériaux provenant de la mobilisation des différents niveaux géologiquement en place. Les premiers remplissages s'accumulent au centre de la structure où ils forment un dôme. Leur composition - limon chocolat à pisolithes et limon argileux gris en alternance - correspond à celle des niveaux profonds et du banc de minerai. Cette dynamique de remplissage montre que la fosse n'a pas été creusée uniformément depuis la surface jusqu'au fond, mais plus vraisemblablement en élargissant un puits. Les mineurs pouvaient travailler en front de taille, dégageant d'abord le minerai à la base, puis abattant la partie supérieure en comblant au fur et à mesure la partie déjà exploitée.

- À l'extrémité sud de la tranchée, la fosse 2.328 n'a pas été repérée sur toute sa largeur. Son bord septentrional 
part en sape sous un bloc de grès, il montre un même élargissement exécuté en reculant la paroi d'un puits.

- F3457 (tranchée $\mathrm{n}^{\circ}$ 12): la fosse mesure 4,40 m de largeur dans l'axe de la coupe. Les premiers comblements sont massés à l'extrémité sud du creusement.

- Immédiatement au nord, la fosse 3452 présente un profil asymétrique: bord vertical au sud, oblique avec un palier dans la partie supérieure, au nord. Une telle déclivité facilite l'accès au fond du puits et la remontée des déblais. Elle est cependant rarement observée. On peut imaginer dans ce cas une fosse inachevée: le creusement, large de 3,50 m en surface, se rétrécit jusqu'au fond. La deuxième étape consistant en l'achèvement de la seconde moitié de la fosse n'a pas été exécutée ici.

Ces différents exemples témoignent de processus de creusement radicalement différents de ceux des puits étroits. Cela illustre un travail en front de taille dans des excavations beaucoup plus vastes. Sans pouvoir être qualifiées de carrières - les plus grandes fosses observées dans les coupes n'excèdent pas $10 \mathrm{~m}$ de large - elles font appel à un travail collectif, tandis que les puits du groupe I peuvent être considérés comme des ouvrages individuels (même si leur multiplication est le fruit de l'activité de plusieurs mineurs).

\section{LE DÉVELOPPEMENT SPATIAL DE L'EXTRACTION}

La carte de répartition montre une organisation concentrique des fosses selon leur type (fig. 35). Les grands creusements du groupe IV sont regroupés dans la zone centrale, à l'exception de F2076, sur la butte stampienne. Les fosses du groupe III se développent essentiellement sur les pourtours de la butte et selon un axe sud-ouest/nord-est correspondant à la pente du terrain; les puits les plus grands se situant dans la partie supérieure. Le groupe II est représenté sur l'ensemble de la surface, avec des regroupements sensibles, notamment dans la partie nord-ouest. Ce phénomène est encore plus flagrant sur le groupe I. Les petits puits sont présents en très grande densité en périphérie de la zone d'extraction où ils forment des concentrations bien délimitées.

L'observation des caractères gîtologiques du minerai montre que l'aire de répartition des grands creusements correspond à la zone où la corrélation profondeur/ densité de minerai assure un rendement optimal de l'extraction. Le groupe I est représenté par trois concen- trations cernant la mine. Le lien entre ces concentrations est par ailleurs renforcé par la morphologie des puits (proportion plus importante de fosses proprement rectangulaires). Cette organisation est en revanche indépendante des caractères gîtologiques du minerai: on retrouve ces puits au sud, près du sommet de la butte, où le minerai est dense mais profond $(-4 \mathrm{~m})$; ils sont également présents au nord où le banc appauvri est à peine à $1 \mathrm{~m}$ sous la surface. Elle peut répondre à un facteur chronologique. Le comportement de ces concentrations vis-à-vis du noyau d'habitat carolingien vient conforter cette hypothèse. Celui-ci est délimité à l'ouest et au nord par un large fossé coudé recoupant les grandes fosses d'extraction placées sur son passage. À l'intérieur, les structures d'habitat (trou de poteau, four domestique, silo...), également postérieures au fosses d'extraction, sont échelonnées entre le $\mathrm{X}^{\mathrm{e}}$ et le $\mathrm{XI}^{e}$ s. Les petites fosses du groupe I se développent très nettement en périphérie de cet enclos. Certaines viennent même mordre sur le fossé nord. Ces concentrations seraient donc vraisemblablement générées par cet habitat tardif, évitant dans un premier temps l'espace habité puis empiétant légèrement dessus (un fond de cabane daté de la fin du $\mathrm{X}^{\mathrm{c}}$ ou du $\mathrm{XI}^{\mathrm{e}} \mathrm{s}$. est recoupé par un puits).

L'hypothèse d'une phase tardive d'extraction se développant en périphérie de la mine s'intègre dans un schéma logique de progression. On peut en effet imaginer que les secteurs assurant le meilleur rendement aient été d'abord exploités intensivement, les marges étant ensuite investies progressivement. Les relations stratigraphiques corroborent ce fait: les grandes fosses centrales sont recoupées par l'enclos carolingien. L'exploitation du versant de la butte, au sud-ouest, est également ancienne: quelques fragments de céramique mérovingienne ont été retrouvés dans trois fosses de ce secteur. Par ailleurs, on note dans cette zone l'apparition de charbons de bois dans les remplissages, sans que l'on puisse la mettre en relation avec l'activité métallurgique (absence de scories, éloignement des structures de combustion liées à la métallurgie). Les rejets charbonneux reflètent ici une activité domestique, attestée par la présence de quelques structures d'habitat: deux ou trois bâtiments sur poteaux, au moins un four domestique. Cette unité d'habitation est datée de la fin du VII ${ }^{\mathrm{c}}$ ou du VIII's.

Un tel modèle de progression implique de prime abord une bonne connaissance du banc de minerai. Des puits de 


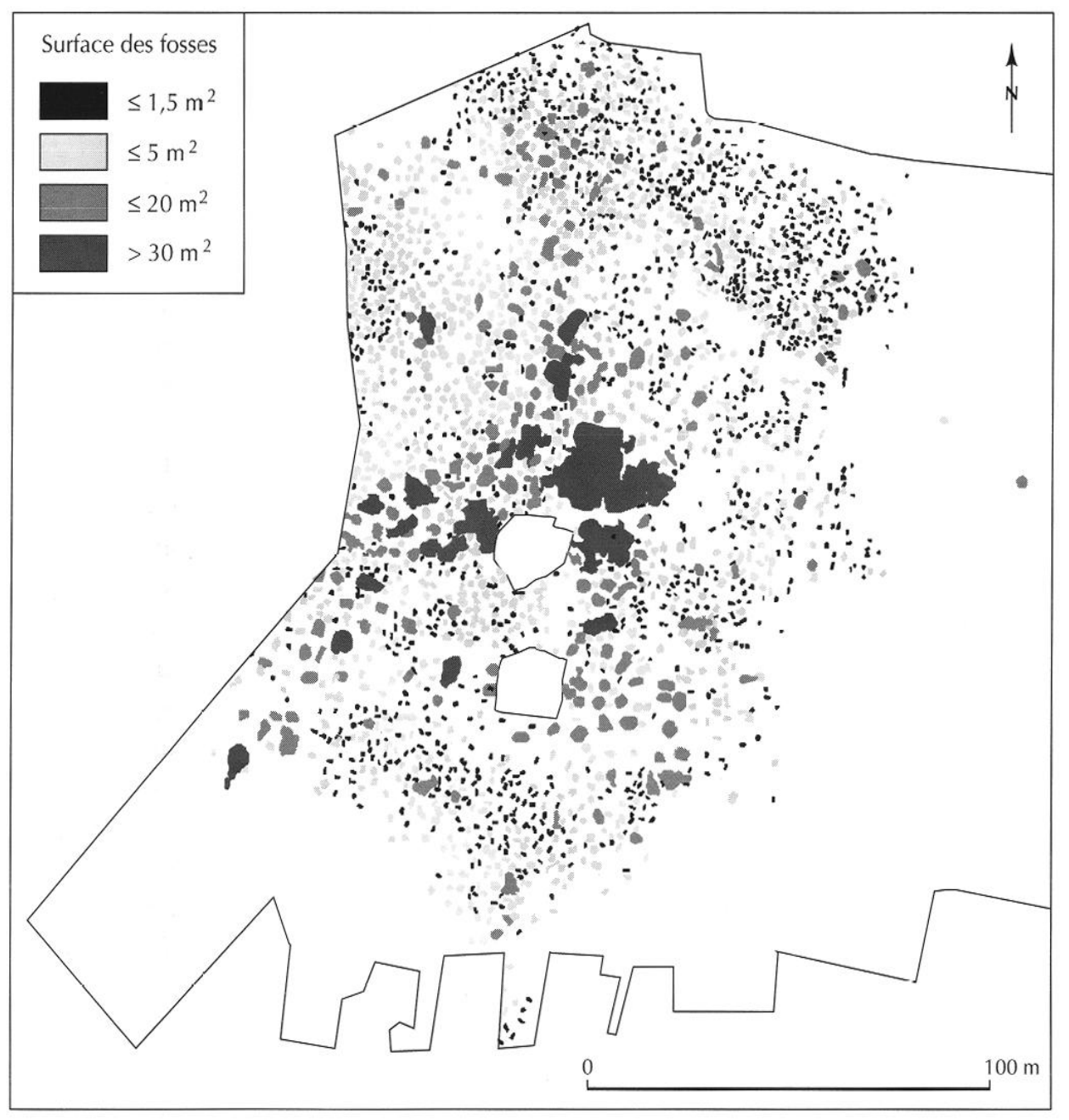

Fig. 35 - I.es Fourneaux à Vert-Saint-Denis (Seine-et-Marne) : plan de la mine. Les fosses d'extraction apparaissent en niveaux de gris en fonction de leur surface en plan.

sondage destinés à évaluer la profondeur et la densité du minerai ont pu être creusés à cet effet. Trois d'entre eux ont été repérés à l'extérieur de la mine, distants jusqu'à plus de $100 \mathrm{~m}$ de la lisière de l'exploitation. Ils n'atteignent pas le minerai, trop profond, et demeurent donc isolés. Ils témoignent de l'action de prospecteurs tentant leur chance parfois au sein même de leur habitat.

Le plan de masse de la mine ne laisse apparaitre que peu de zones vierges. Les sondages mécaniques opérés en ces endroits ont mis en évidence des lacunes dans le banc de minerai. Ces zones ont donc été délaissées sciemment, les fosses alentour s'avérant négatives. Cette observation vient encore appuyer l'image d'une progression graduelle avec creusement des puits de proche en proche, jusqu'à épuisement total d'un secteur.
Les limites de la zone d'extraction sont dictées par la profondeur du minerai, devenu inaccessible (au sud et à l'est), ou par sa raréfaction (au nord).

\section{LES RENDEMENTS}

Deux prélèvements de minerai ont été quantifiés. Le premier se trouve à mi-hauteur de la butte stampienne, sur le banc de minerai non mobilisé, riche en rognons. Il s'agit d'une extraction réalisée dans des conditions assez proches de celles que l'on peut envisager pour l'époque. Le sondage se trouve au cœur de la mine, dans un espace non remanié, situé entre des puits, et qui peut être considéré comme parfaitement représentatif. Ce sondage a été creusé 
au fond d'une tranchée et à partir de l'affleurement de la formation de minerai.

Ses dimensions (1,60 $\mathrm{m}$ de long, $0,70 \mathrm{~m}$ de large et $0,30 \mathrm{~m}$ de profondeur) sont comparables à celles des fosses d'extraction rectangulaires que l'on trouve en grand nombre sur le site.

L'extraction a consisté à piocher l'espace défini et à prélever, au fur et à mesure, tous les nodules de minerai palpables à la main. On atteint ainsi une centaine de kilos de minerai $(\mathrm{sec})$ pour un volume de $0,33 \mathrm{~m}^{3}$ ou $89 \mathrm{~kg} / \mathrm{m}^{2}$.

Le second prélèvement se trouve en bas de pente, au nord. Il touche la couche de limon brun-rouge à pisolithes et concrétions ferrugineuses, beaucoup moins riche que la formation précédente. La valeur extraite est de $41 \mathrm{~kg} / \mathrm{m}^{2}$.

La limite entre les deux formations peut être schématiquement dressée à partir des différentes coupes géologiques. Les deux valeurs de prélèvement leur ont été attribuées.

Considérant que les puits ont des parois verticales, la superficie des fosses en surface du décapage se rapproche de celle atteinte sur le banc de minerai. La somme des surfaces des fosses atteignant la formation la plus riche se monte à $3896 \mathrm{~m}^{2}$. À partir dc la valcur du prélèvement réalisé dans cette zone $\left(89 \mathrm{~kg} / \mathrm{m}^{2}\right)$, on peut en déduire une extraction de l'ordre de $346744 \mathrm{~kg}$ de minerai. En bas de pente, les puits atteignant le banc de minerai remanié totalisent une surface de $2719 \mathrm{~m}^{2}$, soit $111479 \mathrm{~kg}$ de minerai extrait, ce qui donne sur la partie reconnue de la mine une extraction totale de 450 t. Cette estimation comporte bien évidemment une marge d'incertitude: le banc de minerai n'est pas continu et certains puits ne le touchent que partiellement, voire pas du tout. D'autre part, la formation à pisolithes et concrétions ferrugineuses a par endroits été observée au-dessus de celle, plus riche, à rognons de minerai. Il est probable que les deux bancs aient alors été simultanément exploités.

Ces premiers éléments quantitatifs sont importants dans l'estimation des volumes extraits, mais aussi dans les rapports entre la quantité de matière première, les déchets produits et, par déduction, le volume de la production métallique. En tout état de cause, $100 \mathrm{~kg}$ de minerai extraits dans un seul puits constituent une masse importante pouvant alimenter plus d'une réduction. Quant aux grandes fosses du groupe IV, la plupart ont pu livrer plus de $3500 \mathrm{~kg}$ de minerai. Elles peuvent paraître disproportionnées par rapport aux structures de réduction étudiées sur le site.

\section{LE PRÉTRAITEMENT DU MINERAI}

Cette étape est représentée par un peu plus de 80 foyers qui sont situés, dans leur grande majorité, aux abords des trois bas fourneaux implantés au cœur de la mine (fig. 31). Quelques-uns ont été mis au jour à plus de $100 \mathrm{~m}$ au sud-est de la mine et à mi-distance des trois autres ateliers de réduction.

Ces foyers ont des formes assez similaires. Ils ont été aménagés dans des cuvettes de plan ovale ou circulaire, plus rarement rectangulaire. Leurs dimensions sont assez constantes: $0,60 \mathrm{~m}$ de diamètre et $0,20 \mathrm{~m}$ à $0,40 \mathrm{~m}$ de profondeur suivant leur degré d'arasement. Les relations stratigraphiques indiquent une utilisation lors de l'exploitation de la mine. En effet, plusieurs d'entre eux sont recoupés par des fosses d'extraction, tandis que d'autres sont installés dans les couches d'occlusion de ces dernières. L'induration du limon et sa couleur, grisâtre à rouge, provoquées par l'impact thermique, sont irrégulières, le plus souvent superficielles et peu marquées sur le fond. De même, la rareté des fragments de minerai ou des résidus de bois dans le comblement des foyers peut surprendre. Cette rareté suggère que la totalité du minerai grillé mais également les cendres et les charbons de bois étaient prélevés et transportés près du fourneau.

La nécessité d'une calcination préalable s'explique par les caractères du minerai et du gîte. La formation ferrifère se trouve non loin du niveau d'une nappe d'eau. Le niveau phréatique ne semble pas avoir subi de modification très sensible depuis l'époque carolingienne, comme en témoigne la présence de matériaux organiques au fond des puits à eau qui captent cette même nappe. Dans tous les cas, la strate exploitée par les mineurs était très humide et le minerai aggloméré à l'encaissant argileux devait être traité. Deux techniques complémentaires ont pu être mises en œuvre: le lavage et le grillage. Si aucun indice ne permet d'envisager que le minerai ait été lavé, en revanche un séchage par le feu permettait d'éliminer l'eau avec le plus d'efficacité, compte tenu de la structure caverneuse, hétérogène et litée des rognons; en outre, le sédiment chauffé se dissocie naturellement de la roche. 
Il pouvait s'ensuivre un tri, voire un broyage, avant l'introduction du minerai dans le fourneau. Quelques indices semblent se rapporter à ces étapes. Il s'agit de rejets de minerai observés dans le remplissage de fosses d'extraction dans le secteur des foyers de grillage. Ce minerai se présente sous la forme de concentrations de poudre magnétique violacée, comme on en obtient après grillage. Il s'agit très vraisemblablement des rebuts issus du tri du minerai ou de son stockage.

\section{LES ATELIERS DE RÉDUCTION}

I a répartition des concentrations de scories sur le site permet de distinguer au moins six zones de réduction ${ }^{104}$ (fig. 31). Cinq de ces zones ont livré un bas fourneau, tandis que dans la sixième (secteur est) le bas fourneau situé dans un secteur d'occupation très dense n'a pu être trouvé. Celui-ci pouvait être masqué par des remblais ou arasé, voire détruit par les aménagements postérieurs.

Plusieurs de ces zones réservées à la réduction sont situées dans la minière ou sur ses franges. En revanche deux ou trois autres zones se trouvent à plus d'une centaine de mètres de la minière.

Dans aucun des cas, il ne peut être établi de corrélation directe entre le nombre de puits d'extraction et le nombre de bas fourneaux découverts. En effet, d'autres bas fourneaux existent très vraisemblablement dans des secteurs non fouillés, parfois éloignés, comme cela a pu être révélé lors de diagnostics et par quelques observations ponctuelles, à plus de $100 \mathrm{~m}$ au sud des franges de la mine.

\section{LES BAS FOURNEAUX: GÉNÉRALITÉS}

La fouille des structures métallurgiques du site de Vert-Saint-Denis apporte des données totalement inédites sur le fonctionnement de ces petits bas fourneaux, dont le type même était largement méconnu jusqu'alors. Chacun des bas fourneaux a pu être étudié

104. Les concentrations de scories ou leur absence reposent sur des observations réalisées lors des décapages et de l'enregistrement de chacune des structures du site. Cette vision, limitćc à la surfacc, a été complétée par les observations en coupe et les fouilles manuelles. dans le détail. Les couches de comblement ont été fouillées, des plus récentes aux plus anciennes, jusqu'au démontage des différents rechapages. Le lecteur trouvera ci-dessous les données stratigraphiques qui retracent la sédimentation des fours les plus représentatifs.

Ce sont des fourneaux à cuve partiellement enterrée, avec, à l'avant et en contrebas, une fosse dans laquelle les déchets en fusion sont évacués (fig. 36 et 37 ). L'ensemble mesure près de $2 \mathrm{~m}$ de long. La base de la cuve, de plan circulaire et d'une trentaine de centimètres de diamètre, est creusée dans le terrain limoneux sur une profondeur d'environ $30 \mathrm{~cm}$. Des grosses pierres posées de chant marquent l'emplacement de la porte par laquelle la scorie liquéfiée est évacuée, de même que le métal en fin d'opération. Cette porte, qui pouvait être façonnée en limon, recevait un dispositif de ventilation (évent, tuyère en limon et soufflets?), bien que nous n'en ayons pas de témoins directs. Les traces d'impact thermique sur les parois conservées de la cuve et leur asymétrie, de même que la déformation des parois conjuguée à l'emplacement des coulées de scories sont les indices d'une ventilation artificielle. L'emplacement précis de la source d'air pulsé est discuté. Les montants de grès supportaient la partie frontale de la cuve. Son élévation était construite à l'aide de pierres et de limon extraits sur place. Une cuve tubulaire ou tronconique d'au moins $1 \mathrm{~m}$ de hauteur semble envisageable.

Les fours présentent deux variantes d'architecture qui semblent fonction du système d'évacuation des scories utilisé: présence ou non de scories agglomérées dans le fond de la cuve associées ou non à des scories externes en dôme. La variation rencontrée dans les autres scories coulées avec, d'une part, des scories en cordons isolés et, d'autre part, des scories en plaques de plusieurs kilos illustre une gestion différente dans la régulation des évacuations de la scorie, voire des températures plus ou moins élevées suivant les fourneaux.

Le site offre de nouvelles perspectives dans les approches quantitatives et qualitatives. Il s'agit d'études qui n'ont pas pu être développées ou affinées à ce jour, faute de moyen, mais qui pourraient sans doute permettre de caractériser la production métallique et d'approcher la quantité de métal produite. Il convient d'insister sur la prudence qui doit présider à tout essai d'équation entre le volume des déchets, le minerai exploité et le nombre de fourneaux. 
En effet, sur ce type de site il n'est pas possible d'approcher la production à partir du volume de scories. En l'absence de ferrier il est délicat, voire impossible (de nombreuses fosses n'étant pas explorées), d'établir un calcul à partir de la représentation des déchets visibles à la surface des fosses. On ne peut pas non plus se baser directement sur le nombre de fourneaux puisqu'il est probable que plusieurs autres fourneaux existent hors des emprises fouillées. En revanche, le site offre des possibilités intéressantes à partir de l'estimation des quantités de déchets produits pour une réduction.

La mise au jour de coulées de scories en situation primaire est à ce jour quasiment unique et permettra des estimations précises. Rappelons que les premières estimations permettent d'évaluer entre 25 et $30 \mathrm{~kg}$ les scories produites lors d'une réduction. Par ailleurs, l'estimation que nous pouvons faire du volume de minerai exploité est une autre clef dans l'estimation de la production.

$\mathrm{La}$ fouille manuelle de quelques dépotoirs est à ce titre intéressante car elle permet d'esquisser des hypothèses complémentaires. Ainsi deux dépotoirs de scories coulées, conservés dans leur intégralité dans des fosses d'extraction, ont livré plusieurs centaines de kilos de scories coulées. La nature de ces ensembles clos, qui se sont formés en peu de temps, conjuguée d̀ l'analyse stratigraphique des fourneaux, indique que de très nombreuses réductions se succédaient dans le même fourneau. On peut envisager des successions de réductions, très certainement plusieurs dizaines. Ces enchấnements de réductions, que nous appellerons cycles, devaient être entrecoupés de réfections localisées. En effet, les rechapages de paroi mis au jour donnent sans doute une image incomplète de la réalité des réfections qui devaient être beaucoup plus fréquentes mais le plus souvent limitées aux superstructures. Sous l'effet de la dégradation des parois et au terme de plusieurs cycles de réduction, le fourneau devait être très largement reconstruit.

\section{LE PREMIER ATELIER}

\section{Organisation géNÉRALE}

Cet atelier se trouve au nord-ouest du site, au cœur de la mine de fer. Il est possible de distinguer, par la présence de scories, au moins une trentaine de fosses d'extraction contemporaines du fonctionnement de ce bas fourneau. Toutes ces fosses sont situées à l'avant du fourneau, vers l'est ou latéralement, dans un rayon d'une dizaine de mètres. Elles seront colmatées par les déchets issus du four de réduction.

Les fosses les plus proches sont à moins d'une cinquantaine de centimètres de la fosse de coulée du bas fourneau. L'une d'elles, peu profonde, résulte de l'extraction du limon utilisé dans la construction ou dans les réfections de la cuve du fourneau. Quelques trous de poteaux signalent des installations de surface mais aucun plan de construction ne peut être dressé. L'environnement immédiat du four comprend près d'une dizaine de foyers de retraitement du minerai.

Le four est orienté selon un axe est-ouest, le fond de cuve étant situé à l'ouest. La structure mesure $2 \mathrm{~m}$ de long et elle est excavée de $0,25 \mathrm{~m}$ tout au plus dans le substrat limoneux. De la cuve il ne subsiste que la base, circulaire et faiblement excavée dès l'origine. Cet état de conservation ne permet pas de se faire une idée précise de ses transformations successives, que l'on distingue par le nombre de rechapages. Le diamètre de la cuve peut être estimé entre $0,30 \mathrm{~m}$ et $0,40 \mathrm{~m}$ selon les états du fourneau et le fond présente une pente vers l'est. Ce type de disposition favorise l'écoulement de la scorie vers la fosse de coulée.

Une porte sépare la fosse de coulée de la cuve. Son encadrement est constitué de deux blocs de grès de Fontainebleau posés de chant. Ces piédroits assurent le renforcement de la paroi dans une partie fragilisée par l'évacuation intermittente des scories et lors de la récupération de l'éponge en fin de réduction. Ces blocs servent également de support à la partie supérieure de la cuve du côté de la fosse de coulée.

Une rupture de pente souligne le passage du fond de cuve à la fosse de coulée. Cette dernière a un plan ovoïde $(1,50 \mathrm{~m} \times 1,60 \mathrm{~m})$ et un fond plat.

\section{ANALYSE STRATIGRAPHIQUE}

La conservation très partielle de la cuve de ce fourneau constitue une gêne dans la dissociation des phases de fonctionnement. Comme nous l'évoquerons à plusieurs reprises, le dénombrement des phases d'utilisation est approché au mieux à partir d'une distinction des couches de rechapages. Certaines d'entre elles ont d'ailleurs pu totalement disparaître. Par ailleurs, la scorie 
agglomérée dans le fond de cuve peut résulter d'utilisations successives difficilement perceptibles. Ainsi, l'état de conservation de la cuve de ce premier bas fourneau nous a amenés à restreindre le nombre de phases qui se rapportent successivement à la construction de l'appareil, à ses utilisations et à son abandon.

La première séquence (phase 1) correspond à la construction du bas fourneau: creusement des parties basses, mise en place des piédroits et construction de la cuve. Il s'agit sans doute d'une cuve cylindrique de type colonne. Cette cuve est constituée d'une armature de pierres (calcaire et grès) enduite de limon.

À l'exception de quelques traces de rubéfaction, il reste peu d'éléments des premières utilisations de la structure. Lors des séquences suivantes, la porte est modifiée et l'un des piédroits est réinstallé. Une coulée " accidentelle » intervient sans doute lors de la première réduction. Cette coulée, dégagée au moment du démontage du montant sud de la porte, prend naissance dans le fond de cuve, puis elle se faufile à partir d'une fente de retrait du limon entre le bord du creusement de la fosse de coulée et le piédroit. Cette scorie, qui se développe sur toute la hauteur du piédroit, assurait le colmatage de la cuve.

Il y a manifestement une lacune stratigraphique entre cette première coulée et les écoulements retrouvés en place.

Cette lacune s'explique par l'encombrement rapide de la fosse. Elle était régulièrement curée et les scories étaient rejetées dans les fosses environnantes. L'altération thermique du limon dans la fosse de coulée constitue la trace de ces utilisations répétées: le gradient de couleur, du gris bleuté (là où l'impact thermique est le plus fort) au rouge rosé périphérique, marque en effet l'emplacement des écoulements de scories. On visualise assez facilement le cheminement de la scoric cn fusion avec un orifice de coulé décentré vers le sud par rapport au centre de la porte. Le flux était d'abord étroit, puis beaucoup plus étalé dans le bas de la fosse.

Les premiers rejets rencontrés dans la fosse de coulée sont constitués, du côté sud de la fosse, par une couche homogène de cendres et de charbons de bois, résidus du combustible stocké sur le bord de la fosse. À l'inverse, de nombreux fragments de paroi sont concentrés dans l'autre moitié de la fosse. On peut y voir des fragments de la porte, brisée en fin de réduction lors de l'extraction du métal.
Les déchets de la dernière réduction ont été retrouvés en place. Pour la première fois, on dispose d'écoulements complets pour ce type de four. Cet ensemble de scories recourre dcux ćcoulements superposés et nettement distincts, dont le poids peut être estimé entre 25 et $30 \mathrm{~kg}$.

Le premier écoulement mesure une soixantaine de centimètres de long. Il est constitué de deux éléments qui ne sont plus en connexion. Des éléments semblent absents, d'autres sont déplacés dans cette zone d'interruption. La première partie prend naissance au débouché du fond de cuve. Cette amorce verticale, en forme de cordon, traduit la forte pente du haut du glacis de la fosse de coulée. De fines grappes de scories l'entourent. L'essentiel du flux, légèrement dévié latéralement et canalisé dans un creux, rejoint le fond de la fosse de coulée. À cet endroit la scorie forme une pellicule de quelques millimètres d'épaisscur. Cet écoulement est synchrone des résidus de la charge (gouttelettes de scorie, particules de minerai, charbons) accumulés dans le fond du fourneau.

L'autre partie de cet écoulement forme une grosse plaque piégée dans le fond de la fosse de coulée. Ses extrémités sont fragmentées ou incomplètes. Son assise convexe reproduit une petite cuvette ménagée dans les remblais. Le second écoulement est complet. Son amorce, constituée de deux strates, se calque sur la coulée antérieure. Il est moins long $(40 \mathrm{~cm})$ et plus resserré dans ses parties médiane et terminale.

\section{LE DEUXIÈME ATELIER}

\section{ORGANISATION GÉNÉRALE}

Cet atelier est situé à $74 \mathrm{~m}$ au sud-est du précédent bas fourneau. Il se trouve à l'emplacement de la mine, en bordure d'une zone de grandes minières. Les foyers de grillage associés au bas fourneau sont situés plus au nord.

Le bas fourneau présente des similitudes avec le précédent. Il a la même orientation et des dimensions réduites.

On peut dénombrer plusieurs dizaines de fosses d'extraction, qui sont contemporaines de la mise en activité du fourneau ou de peu antérieures. La plupart de ces fosses ont été remplies par des résidus de vidange du four, parmi lesquels on compte de rares fragments de 
paroi. La plupart de ces fosses ont une forme rectangulaire accusée et une profondeur d'au moins 2,50 m par rapport à la surface du décapage. Elles sont disposées de manière concentrique autour du fourneau, avec un espace de dégagement ménagé devant la fosse de coulée. L'une de ces fosses, fouillée manuellement et remplie de scories, est à une cinquantaine de centimètres du fourneau. De l'autre côté du fourneau, une autre fosse, de dimension comparable et tout aussi proche mais exempte de scories, lui semble antérieure.

\section{ANALYSE STRATIGRAPHIQUE}

La structure mesure $1 \mathrm{~m}$ de long et est orientée selon un axe est-ouest. Le fond de cuve est conservé sur une vingtaine de centimètres de profondeur pour un diamètre voisin de $0,25 \mathrm{~m}$ au fond. Ce fond est très légèrement concave. La fosse de coulée ovoïde mesure $1 \mathrm{~m}$ dans sa plus grande largeur; ses bords sont en pente douce. Les aménagements de la porte sont partiellement oblitérés mais leur restitution reste possible par analogie avec le bas fourneau précédent (3001). L'emplacement d'un des piédroits, au sud, subsiste sous la forme d'un négatif d'une trentaine de centimètres de long et d'une dizaine de centimètres de profondeur. Il n'existe pas d'aménagement comparable de l'autre côté de la porte. Selon toute vraisemblance le piédroit a été déplacé, voire supprimé, lors des transformations postérieures. En effet, on constate un déplacement transversal du fond de cuve au cours des utilisations successives. Cette translation du fond de cuve a sans doute eu lieu au moment du réarrangement de la porte du fourneau. Cette cuve initialement située au sud se décale très progressivement vers le nord. Dans son dernier état, la paroi interne du fond de cuve accuse un décalage d'une vingtaine de centimètres par rapport à sa situation d'origine. Ce phénomène est constant dans chacun des fourneaux du site.

Le démontage des parois successives montre qu'il y a eu un regarnissage là où l'impact thermique est le plus prononcé. Les réparations observées à la base de la cuve permettent ainsi de distinguer au moins cinq phases ou cycles de fonctionnement.

À l'instar du fourneau précédent, on retrouve une coulée accidentelle issue des premières utilisations. En amont, cette coulée fait corps avec la paroi d'origine puis elle s'épaissit et se divise en deux cordons qui enserrent le piédroit. Chacune des phases suivantcs comprend une première séquence de construction qui correspond au regarnissage de la paroi et une séquence d'utilisation marquée par l'altération thermique de cette dernière. La scorification du revêtement argileux, d'un aspect très proche de celui des scories coulées, est présente sur toute la hauteur de paroi conservée, mais s'estompe sur le fond, protégé par une pellicule cendreuse. Aucun des dépôts de la fosse de coulée n'est contemporain de ces états.

La phase suivante débute par un nouveau rechapage (construction). Le fond de cuve de plan circulaire est légèrement tronconique $(35 \mathrm{~cm}$ de diamètre en surface et $22 \mathrm{~cm}$ au fond). La paroi la moins altérée (rubéfaction superficielle) est au nord. La couleur passe progressivement du rouge au gris jusqu'à une scorification complète.

La dernière coulée a été trouvée dans sa position d'origine. Elle est constituée de deux écoulements superposés. Le premier, sans doute incomplet, forme une plaque rectangulaire de $0,20 \mathrm{~m}$ par $0,10 \mathrm{~m}$. Il est recouvert par un écoulement figé au passage situé entre la fosse et le fond de cuve. Sa forme est globalement triangulaire avec une amorce large de $0,23 \mathrm{~m}$. Il fait $0,10 \mathrm{~m}$ dans sa plus grande longueur. Dans cette partie, les filets de scorie, nettement dissociés, ont l'aspect d'une résille. Le fond de cuve est recouvert d'une pellicule de grains de minerai calciné et de charbons. Les derniers dépôts illustrent l'abandon de la structure, puis la destruction de la cuve.

Ce type de fourneau semble associé à des scories d'une morphologie sensiblement différente de celles obtenues dans le bas fourneau 3001 . On perçoit ici une prédominance de petites scories en cordons, alors qu'il n'y a pas ou peu de plaques. De même on rencontre quelques scories globulaires jusqu'alors absentes. Elles ont pris la forme du fond de cuve lors de leur refroidissement.

\section{LE TROISIÈME ATELIER}

\section{ORGANISATION GÉNÉRALE}

Il est situé plus à l'est des deux ateliers précédents. Il est constitué d'un bas fourneau installé à même un fossé. Selon les éléments de datation, ce fossé d'enclos remonte à la fin du $\mathrm{V}^{\mathrm{c}} \mathrm{s}$. Cette disposition de l'atelier ne semble pas le fruil du hasard. En effet, le quatrième atelier de 
réduction s'étend sur le côté opposé de cet enclos; une fois encore il y a là un bas fourneau aménagé dans les remblais de comblement du fossé. Ces deux ateliers se développent sur les marges de l'espace enclos. Dans ces deux cas, les structures métallurgiques et les dépotoirs s'organisent longitudinalement dans le fossé ou en deçà.

L'environnement du bas fourneau est constitué de puits d'extraction du minerai dont les plus proches sont à une dizaine de centimètres du bord de la fosse de coulée. La fouille mécanique de plusieurs de ces puits a montré l'absence de rejets de réduction dans la plus grande partie de leur remplissage; seuls les comblements terminaux ont livré des scories. Il s'agit là d'un secteur exploité antérieurement à l'activité de ce bas fourneau où seules les cuvettes, formées par le tassement du sédiment des puits, ont été réutilisées comme dépotoir.

L'une de ces fosses, située à peu de distance de la cuve du bas fourneau, contenait une couche de charbons de bois et de minerai, représentant les reliquats d'une charge. Une datation ${ }^{14} \mathrm{C}$ de ces charbons situe ainsi l'activité de ce bas fourneau vers le milieu du VIII' $s$.

La structure est orientée d'est en ouest (fig. 36a). À la différence des autres bas fourneaux, la cuve est située à l'est. Le fourneau présente une forme et des proportions semblables aux structures précédentes avec, d'une part, un fond de cuve dans un bon état de conservation, de $0,25 \mathrm{~m}$ de diamètre et de profondeur, et, d'autre part, une fosse de coulée oblongue $(1,50 \mathrm{~m} / 1,20 \mathrm{~m})$ aux parois obliques.

\section{ANALYSE STRATIGRAPHIQUE}

La succession des dépôts peut être ordonnée en trois phases avec une subdivision en séquences de construction et d'utilisation pour les deux premières. La troisième phase reflète la destruction et l'abandon de la structure. Il ressort de son examen que le fonctionnement est assez bref. Cet aspect est corroboré par le volume des rejets de réduction, qui est moindre que celui des ateliers précédents.

La configuration initiale de l'appareil peut être restituée, même si la paroi d'origine n'a pas été dégagée, en raison du prélèvement de ce bas fourneau qui pourrait faire l'objet d'une présentation dans un musée.

Si la plupart des premiers aménagements sont oblitérés par la reconstruction du fourneau, il est néanmoins possible, à partir de la courbure du segment de cuve préservé sur le côté nord du bas fourneau, de restituer une cuve de plan circulaire d'une trentaine de centimètres de diamètre interne. La porte est délimitée (au nord) par un pilier en grès de Fontaineble'du. La paroi de la base de la cuve prend appui sur ce grès.

Aucun des déchets collectés dans l'appareil ne peut être mis en rapport avec sa première phase de fonctionnement. Seule l'altération thermique des parois s'y rattache. Le gradient de couleur est accusé, avec un premier cerne grisâtre de $0,06 \mathrm{~m}$, qui se développe à partir de la face interne de la cuve, puis un second rubéfié de $0,06 \mathrm{~m}$ à $0,08 \mathrm{~m}$.

La deuxième phase de fonctionnement débute par la reconstruction de l'appareil avec des transformations de son architecture. On remarque un déplacement transversal et longitudinal de la cuve. L'ancienne base du fourneau est partiellement détruite lors de ces aménagements. Cette reprise affecte également la fosse de coulée, qui est élargie au niveau de la porte.

La partie enterrée de la cuve a une forme cylindrique de $0,25 \mathrm{~m}$ de diamètre, tant à la base qu'à son niveau supérieur. La paroi latérale (à l'ouest) est désaxée et rentrante dans sa partie inférieure, tandis que le fond de cuve est très légèrement concave et présente des traces d'arrachement. L'altération la plus prononcée se trouve à la hauteur de l'arasement de la cuve et plutôt à l'ouest. À cet emplacement la paroi est totalement vitrifiée et scorifiée. Ces marques tendent à s'atténuer vers le fond, où elles sont remplacées par une altération noire plus superficielle. Selon nos premières hypothèses, la concavité dans la paroi de la cuve résulte de la fusion du limon et/ou de l'arrachement de la scorie piégée dans le fond $\mathrm{du}$ four, voire de l'éponge de fer. Cette déformation permet d'expliquer le retrait systématique des différentes cuves et leur rechapage. Un petit bloc de grès, à surface horizontale, est intćgrć à la paroi de la cuve là où la chaleur était la plus intense.

Les déchets retrouvés dans ce fourneau sont issus d'une seule opération de réduction. Deux écoulements ont été retrouvés en situation primaire dans la fosse d'évacuation.

Le premier repose au contact du limon du fond de la fosse. Cette plaque de scorie mesure $0,40 \mathrm{~m}$ de long et $0,26 \mathrm{~m}$ dans sa plus grande largeur et elle pèse près de $5 \mathrm{~kg}$. Son épaisseur atteint $0,35 \mathrm{~m}$ au centre et $0,07 \mathrm{~m}$ à $0,08 \mathrm{~m}$ sur les côtés. La source de l'écoulement est située contre le bord sud de la porte. Bien que l'amorce soit 


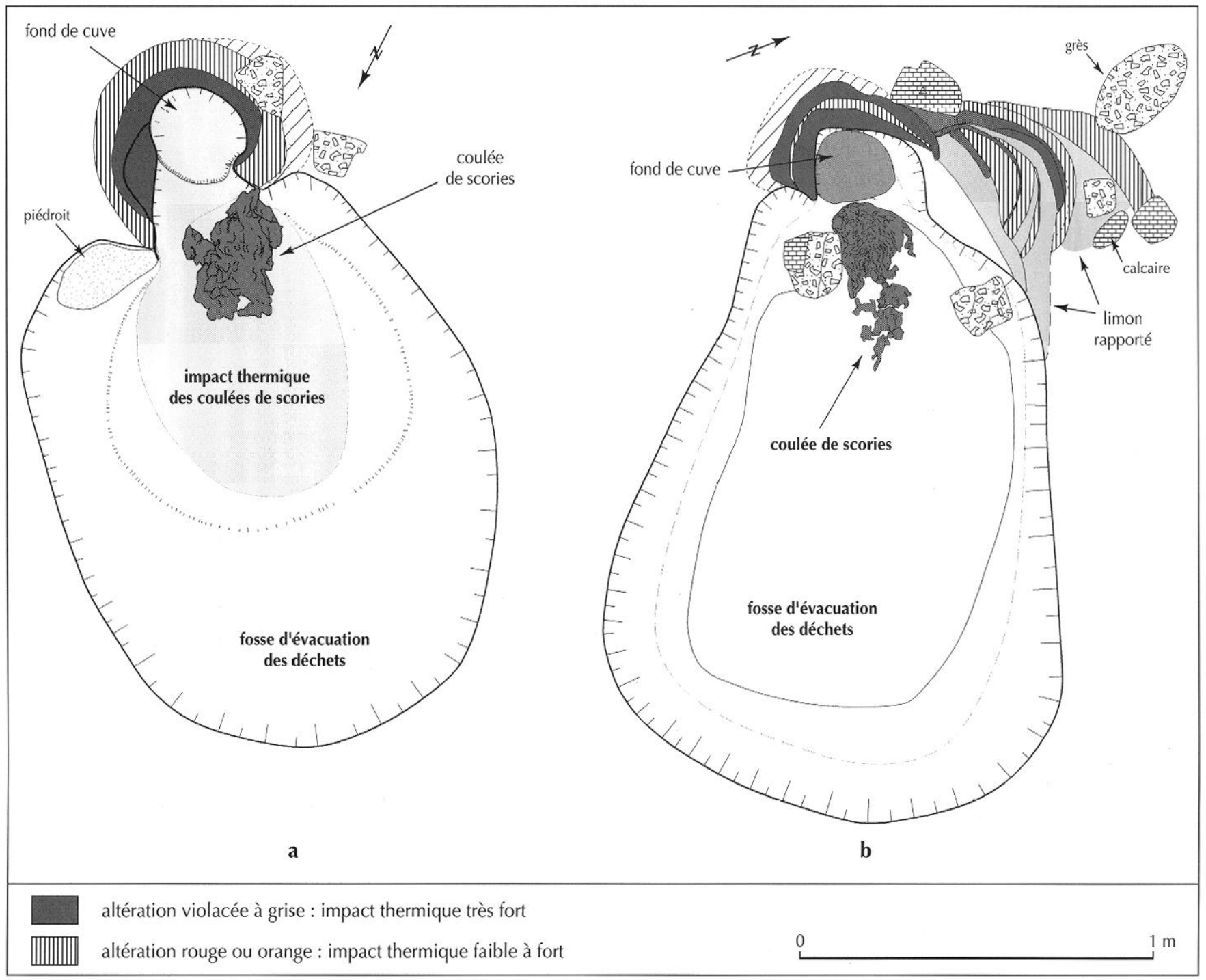

Fig. 36 - Les Fourneaux à Vert-Saint-Denis (Seine-et-Marne) : plan des derniers états des bas fourneaux 10020 (a) et 5712 (b). Sur ce dernier, le déplacement latéral de la cuve est bien visible (dessin V. Goustard).

incomplète, la coulée est, à cet cndroit, trc̀s rcsscrrćc ct l'ouverture pratiquée dans la porte était voisine de $0,04 \mathrm{~m}$ de diamètre. La face antérieure de la scorie a un profil en « $V$ ».

Le dernier écoulement est plus important. Le dépôt forme une grosse plaque qui reprend l'emplacement de la précédente. Cette superposition indique un intermède assez bref entre les deux coulées. Cette coulée mesure $0,65 \mathrm{~m}$ de long et $0,30 \mathrm{~m}$ de large. Elle pèse un peu plus de $16 \mathrm{~kg}$. Si l'on tient compte des lacunes des extrémités, on peut estimer sa longueur initiale entre $0,70 \mathrm{~m}$ et $0,80 \mathrm{~m}$ de long.
Les dépôts accumulés sur le fond du fourneau sont synchrones des coulées. Ils sont constitués d'une couche cendreuse, de petites scories coulées globuleuses et de particules de minerai.

\section{LE QUATRIÈME ATELIER}

\section{ORGANISATION GÉNÉRALE}

Cet atelier est situé à environ $80 \mathrm{~m}$ au sud-est des franges de la minière. Le bas fourneau 5712 , orienté 


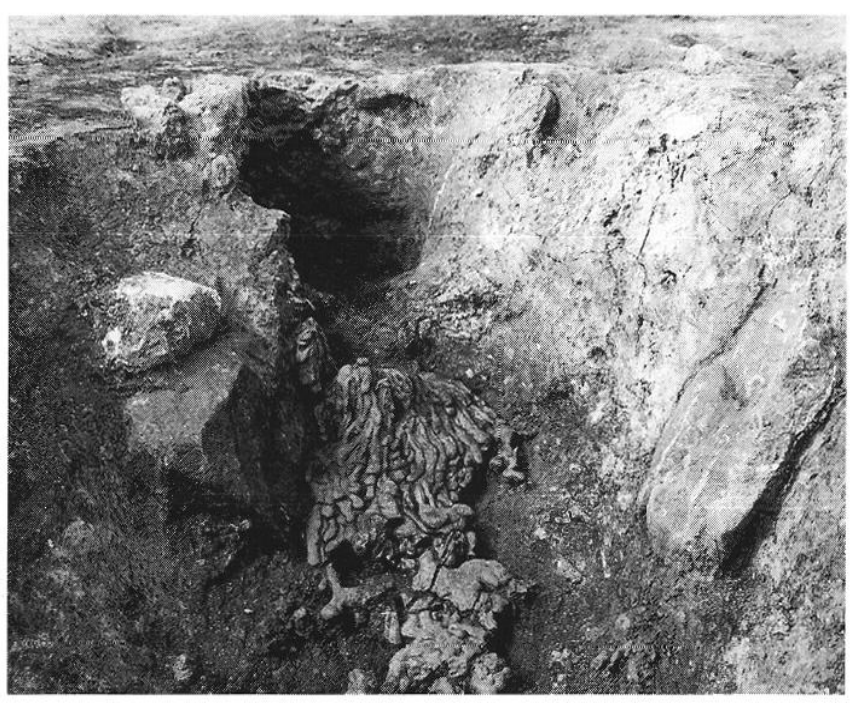

Fig. 37 - Les Fourneaux à Vert-Saint-Denis (Seine-et-Marne), le bas fourneau 5712 : vue frontale de la muspe et de la dernière coulée de scorie en place (cliché AFAN).

selon un axe est-ouest, est creusé dans un fossé. Ce four jouxte, au nord-est, un important ensemble de fosses d'extraction de limon partiellement réutilisées comme dépotoirs. On peut associer à cet ensemble un petit foyer (de charbonnage?) situé à $5 \mathrm{~m}$ vers le nord et creusé dans le fossé. À l'opposé, nous avons mis au jour une concentration de poudre de minerai dans une cuvette. Cette structure, subrectangulaire et d'environ $1,30 \mathrm{~m}$ de long, était comblée par une couche de cendres sur laquelle reposait un dépôt de minerai grillé. Il peut s'agir d'un foyer, mais il n'y a pas de preuve d'une combustion sur place; il peut s'agir d'un espace de stockage.

Les zones de rejets de scories sont assez éparses. Une vue d'ensemble de ce secteur révèle un déséquilibre entre la représentation des déchets et la longévité du fourneau confirmée par le nombre élevé de rechapages. Ce déséquilibre et la présence de nombreuses scories dans les terres de labours à cet endroit suggèrent l'arasement d'un ferrier.

La disposition et les proportions du bas fourneau 5712 sont très similaires à celles des fourneaux précédents (fig. 36b et 37). Sa cuve est partiellement enterrée et la fosse de coulée mesure environ $1,70 \mathrm{~m}$ de long et $1,40 \mathrm{~m}$ dans sa plus grande largeur. Selon les différents états de la construction, la cuve est conservée entre $0,20 \mathrm{~m}$ et $0,25 \mathrm{~m}$ de profondeur et le fond est nettement concave. Son profil est tronconique avec des diamètres de $0,15 \mathrm{~m}$ à la base et de $0,30 \mathrm{~m}$ à $0,40 \mathrm{~m}$ au niveau de son arasement.

Ce sont en fait dix fourneaux qui se sont succédé au même emplacement. Dans l'ensemble, leur état reste fragmentaire. Il subsiste en général une partie de la paroi située au nord et les dépôts de charbons accumulés dans le fond. Dans son état initial, le fourneau est situé au nord et les constructions successives se décalent progressivement vers le sud. Ces écarts successifs proviennent de la déformation de la paroi.

\section{ANALYSE STRATIGRAPHIQUE}

La première phase comprend la construction de l'appareil et ses premières utilisations. Il reste très peu d'éléments de la construction primitive: quelques traces de rubéfaction et quelques blocs de grès sans doute intégrés au soubassement de la cuve. La fouille du fond de cuve a montré la superposition de deux couches cendreuses, entre lesquelles s'intercale une pellicule de limon. Le bord nord de la fosse de coulée est conservé, ainsi que l'aire de coulée où l'altération, due aux écoulements successifs, est très prononcée. Le limon, cuit au contact de la scorie en fusion, forme une croûte grise de $0,70 \mathrm{~m}$ de long et $0,20 \mathrm{~m}$ de large. À son contact, ont été dégagés de petits tronçons de scories et parallèlement des charbons de bois recouvrent le fond de cuve. La fosse est ensuite recouverte de dépôts remaniés constitués de scories coulées et de morceaux de terre cuite.

Les phases suivantes regroupent une alternance de couches de construction (rechapage) et d'utilisation. Certains de ces rechapages intègrent de nombreux moellons de calcaire. Ils peuvent nous donner une idée de la superstructure de la cuve du fourneau. Au cours de ces différentes phases, l'emplacement des coulées évolue peu et très progressivement. L'impact thermique de ces dernières est peu marqué, car elles s'écoulaient sur les remblais sous-jacents.

Les derniers états sont plus intéressants parce que la structure du four est plus complète. Cette phase débute par un réaménagement important. Le fourneau est très nettement décalé transversalement et la fosse de coulée est agrandie du même côté. Des blocs de grès sont utilisés comme piédroits. Chacune des phases suivantes s'individualise par un regarnissage de la paroi. Le maximum d'intensité thermique est toujours très localisé. 
La dernière phase débute par une réfection de la paroi. Un enduit de limon sableux est apposé sur le pourtour du fond de cuve et vient recouvrir partiellement les piédroits. La fosse de coulée contient une multitude de scories, généralement de petite taille, qui se rapportent aux premiers écoulements. On notera, au bas du "glacis ", la présence d'une scorie en dôme. Ces dépôts sont remaniés par les manipulations postérieures.

La dernière coulée demeurait dans sa position d'origine. Elle est synchrone des dépôts du fond de cuve: scorie grappée solidaire de la paroi et couche de charbon de bois. Cet agglomérat, assez friable, est localisé dans l'angle ouest. Il est à environ $8 \mathrm{~cm}$ au-dessus de la base du fourneau.

L'écoulement retrouvé en place se différencie par sa forme ramassée des scories qui ont coulé à l'extérieur $\mathrm{du}$ fourneau et qui se sont refroidies sous la forme de plaques ou de cordons. Dans le cas présent la scorie forme un dôme en contrebas du trou d'écoulement. Son amorce est plaquée contre le piédroit sud. Elle forme une grappe verticale de $0,02 \mathrm{~m}$ à $0,03 \mathrm{~m}$ de diamètre. Le corps principal de la scorie est à $0,06 \mathrm{~m}$ en contrebas de l'amorce. L'ensemble de l'écoulement mesure environ une cinquantaine de centimètres de long pour un poids de $5 \mathrm{~kg}$. Ce type de fourneau se distingue aussi par un fond de cuve de forme nettement concave. Cette forme et le mode d'évacuation des déchets déterminent l'existence de scories de morphologie différente de celles des fourneaux précédents. Il y a, d'une part, une accumulation au fond du four et, d'autre part, une évacuation depuis un point haut du surplus dans la fosse de coulée. Les scories de fond de cuve sont extrêmement abondantes dans tous les dépotoirs de la partie méridionale du site et se distinguent assez aisément par leur morphologie des culots d'épuration. Une étude descriptive de leur structure et de leur composition chimique permettra de dégager leurs caractères propres. Le poids de ces pièces varie d'un peu plus de $1 \mathrm{~kg}$ jusqu'à plus de $2,5 \mathrm{~kg}$.

Les dépôts qui viennent d'être décrits sont scellés par l'effondrement d'une partie de la cuve qui était constituée de nombreux moellons de calcaire.

Nous n'aborderons pas l'étude détaillée du dernier fourneau dans la mesure où il n'apporte pas d'élément supplémentaire du point de vue technologique. La base de la cuve a totalement disparu, mais il subsiste le fond de la fosse d'évacuation avec deux grosses plaques qui semblent être en place. Sa situation reste néanmoins intéressante dans l'approche spatiale et chronologique du site.

$$
*
$$

\section{DES TECHNIQUES DE PRODUCTION ENCORE MÉCONNUES}

La fouille du site des Fourneaux apporte une vision nouvelle de la métallurgie du fer au haut Moyen Âge. Son intérêt est majeur pour l'histoire des techniques puisqu'il offre une chaîne opératoirc continue depuis l'extraction jusqu'à la réduction. L'approche de ce type de mine et des techniques de traitement du minerai correspondantes n'a à ce jour pas d'équivalent en France. La conservation, exceptionnelle, dans les fosses de rejet des bas fourneaux, des coulées de scories provenant de la dernière réduction permettra de connaitre le volume de déchets produit par une telle opération. Le calcul du minerai exploité et des volumes respectifs des scories produites nécessite un complément d'étude. La poursuite de l'exploitation de ces données permettra d'établir un réfërentiel pour l'estimation des rapports minerai-déchets-métal, et d'estimer la production métallique d'un tel site. On peut supposer, selon les premiers éléments et pour fixer un ordre de grandeur, que le minerai collecté dans une fosse d'extraction du module le plus courant alimentait une à trois réductions et qu'une opération permettait de produire une dizaine de kilos de métal, voire un peu plus.

\section{UN SITE ARTISANAL INTÉGRÉ DANS UNE ÉCONOMIE DOMANIALE?}

La présence d'un habitat associé constitue l'un des intérêts fondamentaux du site en nous permettant d'intégrer cette activité dans son cadre social. L'habitat des Fourneaux ne diffère en rien, à première vue, des sites ruraux voisins. L'agriculture y est pratiquée, ainsi que les autres occupations domestiques recensées sur les sites voisins. Il conviendra d'estimer l'importance de ces activités. Six siècles d'aménagements du haut Moyen Âge provoquent une impression de densité d'occupation 
qu'il faut relativiser. L'absence de nécropole, notamment, nous conduit à qualifier cet habitat de hameau plutôt que de village. L'étude des biens de consommation, en particulier de l'alimentation carnée, devrait contribuer à distinguer d'éventuelles spécificités dans le mode de vie et le statut des habitants et à évaluer l'impact économique et social de la métallurgie. Malgré l'ampleur des structures liées à cet artisanat, l'impression domine d'une exploitation d'importance plutôt limitée, en raison de la longue durée de son fonctionnement. L'organisation des ateliers et le regroupement des foyers de traitement semblent illustrer une division du travail. L'activité métallurgique n'a cependant pu concerner qu'un nombre restreint d'individus. L'extraction, en particulier, repose pour une grande part sur un mode individuel de travail.
Quelle est donc la part de la métallurgie dans l'économie de cet habitat? S'agit-il de sa ressource principale, ou, au contraire, d'une occupation complémentaire, voire saisonnière?

Aucune trace tangible de mise en forme du métal ou d'élaboration d'objet n'a été mise en évidence dans les limites de la zone fouillée. Il faut vraisemblablement envisager une exportation du métal vers le centre urbain de Melun ou vers d'autres établissements dépendant d'une même unité domaniale. Dans un rayon de quelques kilomètres, plusieurs sites fouillés récemment sur l'autoroute A5 ont livré des indices de forgeage pour la même période. Si l'on ne peut établir avec certitude un lien direct entre ces établisscments, il cst pcrmis de s'intcrroger sur les réseaux de distribution et la spécialisation des habitats du haut Moyen Âge. 